\title{
Nonlinear thermal effects in unsteady shear flows of a rarefied gas
}

\author{
Y. Ben-Ami and A. Manela* \\ Faculty of Aerospace Engineering, Technion-Israel Institute of Technology, Haifa 32000, Israel
}

(Received 10 July 2018; published 28 September 2018)

\begin{abstract}
We study the response of a rarefied gas in a slab to the motion of its boundaries in the tangential direction. Different from previous investigations, we consider boundaries displacements at nonsmall Mach (Ma) numbers, coupling the dynamic and thermodynamic gas states, and deviating the system from its low-velocity isothermal condition. The problem is studied in the entire range of gas rarefaction rates, combining limited case ballistic- and continuum-flow analyses with direct simulation Monte Carlo computations. A nonlinear solution is derived in the ballistic regime for arbitrary velocity profiles and amplitudes. At near-continuum conditions, a slip-flow timeperiodic solution is obtained for the case of oscillatory boundary motion, by expanding the flow field in an asymptotic Mach power series. The effect of replacing between isothermal and adiabatic surfaces is examined. The results indicate that, at all Knudsen (Kn) numbers, the thermodynamic fields and normal velocity component are dominated by double-frequency (and descending higher-order even-frequency harmonic) time dependence, different from the fundamental-frequency time dependence dominating the tangential gas velocity. At continuumlimit conditions, this stems from the quadratic viscous dissipation term (negligible at low-Mach conditions), coupling the square of the tangential velocity gradient as a forcing term. System nonlinearity also results in an unsteady normal force acting on the boundaries, overcoming the tangential force with increasing Ma. A marked difference from the latter is that the normal force either decreases with $\mathrm{Kn}$ or, at sufficiently small actuation frequencies, varies nonmonotonically with $\mathrm{Kn}$, reaching a maximum at some intermediate rarefaction conditions.
\end{abstract}

DOI: 10.1103/PhysRevE.98.033121

\section{INTRODUCTION}

The flow field generated by the motion of a planar boundary in its tangent direction is a classical problem in viscous fluid dynamics that has been studied extensively over the years [1]. Considering a canonical setup of an impulsively started boundary (Rayleigh's problem), or harmonically displaced surface (Stokes' second problem), the system response was analyzed, yielding the transient and final states of the fluid, as well as the shear force acting on the solid wall. Under continuum incompressible conditions, the dynamic flow state becomes decoupled from its thermodynamic description, resulting in a flow field where the thermodynamic distributions of density, temperature, and pressure are uniform. The tangential velocity field is then governed by a single momentum balance in the tangent direction.

While incompressible flow conditions prevail at relatively low boundary velocities, fluid compressibility becomes significant when the surface-based Mach number increases. To consider this problem, several works have analyzed Rayleigh's problem for a viscous compressible gas, combining earlyand late-time approximations with boundary-layer-type solutions for the propagating disturbance [2-5]. The works have demonstrated the coupling between the flow dynamic and thermodynamic properties at nonsmall velocities, originating from viscous dissipation effects. When considering the earlytime response of the system, it was argued that a rigorous analysis of the rapid changes observed at the wall surface

*amanela@technion.ac.il requires reconsideration of the problem, taking into account the gas microscopic (molecular) characteristics [2].

When the characteristic length- or timescale of the flowfield become comparable with the gas molecular mean free path or time, respectively, the continuum description breaks down, and the microscopic properties of the medium must be modeled. In the context of boundary-induced shear flows, such conditions may occur in either a narrow slab, where the gas is confined to a thin layer compared with the mean free path, or when boundary-induced motion is characterized by exceedingly short timescales, as in Rayleigh's problem at short times, or in Stokes' second problem at high actuation frequencies. Adopting the Bhatnagar, Gross, and Krook (BGK) kinetic model, Cercignani and Sernagiotto [6], Sone [7], and Trilling [8] studied the linearized Rayleigh problem for a rarefied gas in a semi-infinite domain. Later works have then considered shear-flow problems in confined setups, to address the steady Couette flow. Among others, Beskok, Karniadakis, and Trimmer [9] investigated the compressible problem in the slip-flow regime, demonstrating the nonlinear effect of large boundary velocity in deviating the density and temperature fields. A comprehensive overview of the steady Couette problem was presented by Garzó and Santos [10], allowing for both tangential velocity and temperature differences between the boundaries, and comparing between kinetic models predictions and Monte Carlo-based simulation results. The counterpart time transient response to an impulsively shifted surface was considered in other works, including Stefanov, Gospodinov, and Cercignani [11], and Abramov and Butkovskii [12]. Basing their analyses on numerical simulations, wall velocities at arbitrary Mach numbers were examined. 
In a separate set of studies, the effect of gas rarefaction on the wall-induced unsteady Couette flow was investigated. Beskok and coworkers $[13,14]$ analyzed the linearized problem for small-amplitude harmonic oscillations and developed an empirical model to approximate the system low-frequency response. Hadjiconstantinou [15] reconsidered the problem and applied a second-order slip-flow model to capture the gas behavior in the transition regime. These works were complemented by Refs. [16-18], which analyzed the transition between the continuum and ballistic limits for a BGK [16] and hard-sphere $[17,18]$ gases in the entire range of oscillation frequencies and Knudsen numbers. Considering geometrical setups other than a planar slab, Gospodinov, Roussinov, and Stefanov [19] investigated the oscillatory Couette flow between concentric cylinders by applying a continuum-limit finite-difference scheme at low Knudsen numbers. Wu, Reese, and Zhang [20] addressed the low-amplitude oscillatory flow developed in a two-dimensional cavity with an harmonically displaced lid.

Different from existing rarefied gas studies on the steady Couette problem, most of the above-mentioned unsteady flow investigations [13-18,20] have focused on the limit of small boundary velocities, thus neglecting the inevitable effect of thermal coupling at high surface speeds. In the few cases where nonsmall surface speeds were considered, numerical computations were carried out. Thus, Gospodinov et al. [19] studied the nonlinear cylindrical Couette problem in the continuum limit using a finite-difference scheme. More recently, Wang et al. [21] investigated the two-dimensional problem in a rectangular cavity based on numerical discretization of the Shakhov kinetic equation. To gain further insight into the effects of gas rarefaction and system nonlinearity, the present work focuses on the unsteady Couette problem at nonsmall wall velocities in a planar one-dimensional setup. The relatively simplified configuration facilitates the calculation of analytic approximations for the collisionless and continuum gas responses, which, in turn, shed light on the coupling mechanisms between the dynamic and thermodynamic gas states at different rarefaction levels.

Apart from the fundamental interest in studying the above problem, specific applications exist. In particular, situations where nonsmall Mach number flows appear at noncontinuum conditions have recently become of practical significance for the design of high-speed vehicles, including high-altitude stratospheric platforms [22] and ground-level machines. In the latter, high-speed shear flows are encountered in the Hyperloop machine [23], a sealed tube through which a pod vehicle at near-vacuum conditions is set into motion. At the low densities considered, rarefied flow conditions prevail, accompanied by significant shear rates over the vehicle surfaces. Transonic speeds are considered (with a Mach number approaching $\approx 0.9$ ), and the flow field turns inevitably unsteady during acceleration or deceleration of the body at entrance and departure. Several investigations on rarefied transonic gas flows over airfoil-shaped surfaces have been carried out, specifically for the Hyperloop configuration [24,25], as well as for examining the coupling between the dynamic and thermodynamic gas states (e.g., Refs. [26,27]). The understanding of the gas behavior in this regime is a key element in the above developments, and we consider the present contribution as a step towards such analysis. Considering other applications, relatively high-speed shear flows at rarefied conditions prevail in vacuum pump machines, such as the Holweck pump, where flow Mach numbers as high as $\approx 0.5$, and Knudsen numbers reaching $\approx 0.5$, are reported [28-30]. Several works [21,31] have mentioned the relevance of the nonlinear Couette problem for the analysis of microelectromechanical systems operating at high $(\approx 100 \mathrm{GHz})$ frequencies and oscillation amplitudes of several nanometers.

We consider a planar one-dimensional bidirectional setup of a gas in a slab, where the confining walls are displaced in their tangential direction and kept at fixed thermal conditions. The study combines analytical and asymptotic solutions in the ballistic and continuum limits with direct simulation Monte Carlo (DSMC) predictions. At first, an analytic solution for the collisionless gas response to arbitrary wall-shear excitation is obtained. Focusing on the case of oscillatory boundary motion, the results illustrate the effect of replacing between temperature-fixed (isothermal) and heat-fluxfixed (adiabatic) surfaces. It is shown that nonlinear effects, originating from nonsmall actuation velocities, give rise to spatially nonuniform thermodynamic deviations, dominated by double-frequency periodic time variations and higher-order even-frequency harmonics. These nonuniformities, apart from affecting the tangential shear stresses at the walls, result in normal surface loading, not encountered in the linearized isothermal approximation. The results obtained in the large and small Knudsen number limits are validated using DSMC calculations, and the conditions where the limit-case analyses break down are discussed.

An outline of the paper follows. In the next section, the general problem is described. The ballistic and continuum limits are analyzed in Secs. III and IV, respectively. The direct simulation Monte Carlo method, applied for problem analysis at arbitrary rarefaction conditions, is discussed in Sec. V, where a noniterative algorithm for the implementation of a heat-flux boundary condition is specified. Our results are presented in Sec. VI, followed by conclusions in Sec. VII. Technical details regarding the ballistic and continuum solutions are relegated to the Appendices.

\section{PROBLEM STATEMENT}

Consider a layer of a monatomic hard-sphere gas confined between infinite parallel fully diffuse walls at $y^{*}=-L^{*} / 2$ and $y^{*}=L^{*} / 2$ (with asterisks denoting dimensional quantities). The wall at $y^{*}=L^{*} / 2$ is set at fixed temperature, while the wall at $y^{*}=-L^{*} / 2$ is set at either isothermal or adiabatic (thermally insulated) conditions. The gas is initially at rest with uniform density $\rho_{0}^{*}$ and temperature $T_{0}^{*}$, and is in thermodynamic equilibrium with the boundaries. At time $t^{*} \geqslant 0$, the walls are displaced in the tangential $\hat{\mathbf{x}}$ direction, according to

$$
\mathbf{U}_{\mathbf{w}_{1,2}}^{*}\left(t^{*}\right)=U_{w_{1,2}}^{*}\left(t^{*}\right) \hat{\mathbf{x}}
$$

where the subscripts $w_{1}$ and $w_{2}$ correspond to the properties of the $y^{*}=-L^{*} / 2$ and $y^{*}=L^{*} / 2$ walls, respectively. Additionally, the $y^{*}=-L^{*} / 2$ boundary is set at either isothermal 
or adiabatic conditions,

$$
T_{w_{1}}^{*}=T_{0}^{*} \quad \text { or } \quad \mathbf{Q}_{\mathbf{w}_{\mathbf{1}}}^{*}\left(t^{*}\right) \cdot \hat{\mathbf{y}}=0
$$

respectively, while the $y^{*}=L^{*} / 2$ boundary is isothermal,

$$
T_{w_{2}}^{*}=T_{0}^{*} .
$$

In the following, we analyze the gas response to the tangential-velocity disturbances in Eq. (1), in conjunction with the different sets of thermal conditions in Eqs. (2) and (3). Imposing a heat flux condition on only one of the walls, while keeping the other wall isothermal, ensures that the gas thermal energy cannot increase infinitely, and a final state with finite hydrodynamic deviations is obtained. A study on the effect of thermal conditions on the system response may then be carried out.

Considering the system behavior at nonlinear conditions, the input velocity is taken nonsmall, and of an order equivalent to a unity Mach number (see Eq. (5) et seq.). For each specific choice of the boundary conditions, semianalytic results are obtained in the collision-free and continuum limits, supplemented by direct simulation Monte Carlo predictions. The latter are used for validation of the limit-case results and for evaluating the gas response in the intermediate range of gas rarefaction. While the analysis is carried out in part for an arbitrary choice of the signal walls inputs $U_{w_{1.2}}^{*}\left(t^{*}\right)$, our results focus on the case of harmonic counter-phase walls oscillations,

$U_{w_{1}}^{*}\left(t^{*}\right)=\bar{U}_{w}^{*} \cos \left(\omega^{*} t^{*}\right) \quad$ and $\quad U_{w_{2}}^{*}\left(t^{*}\right)=-\bar{U}_{w}^{*} \cos \left(\omega^{*} t^{*}\right)$,

where $\bar{U}_{w}^{*}$ and $\omega^{*}$ denote the walls common velocity amplitude and displacement frequency, respectively. The choices of equal amplitudes and counter-phase motions enable comparable study of the forces acting on the boundaries, as well as maintaining the gas at maximal shear rate. While the effect of walls-motion phase difference on the system response may be readily studied, our results indicate that it does not yield any new phenomena that are not encountered in the opposing motion setup, and therefore omit its discussion. The analysis of the harmonic problem may be treated as a Fourier decomposition investigation of a more general (possibly nonperiodic) actuation input.
To proceed to problem analysis, variables nondimensionalization is introduced. We normalize the length and velocity by the gap width $L^{*}$ and the mean thermal speed $U_{\mathrm{th}}^{*}=\sqrt{2 \mathcal{R}^{*} T_{0}^{*}}$, respectively, where $\mathcal{R}^{*}$ denotes the specific gas constant. The time is normalized by $L^{*} / U_{\mathrm{th}}^{*}$, and the density and temperature are scaled by $\rho_{0}^{*}$ and $T_{0}^{*}$, respectively. The pressure is normalized by $\rho_{0}^{*} U_{\mathrm{th}}^{* 2}$, and the heat-flux is nondimensionalized by $\rho_{0}^{*} U_{\mathrm{th}}^{* 3}$. For the case of harmonic excitation, the scaled problem is governed by the parameters

$$
\mathrm{Kn}=\frac{l^{*}}{L^{*}}, \quad \mathrm{Ma}=\frac{\bar{U}_{w}^{*}}{U_{\mathrm{th}}^{*}}, \quad \text { and } \quad \omega=\frac{\omega^{*} L^{*}}{U_{\mathrm{th}}^{*}},
$$

denoting the gas Knudsen number, thermal-velocity-based Mach number, and nondimensional frequency, respectively, with $l^{*}$ marking the mean free path of a gas molecule. As noted above, we focus on the gas response to walls actuation at nonsmall Mach numbers in the entire range of Knudsen numbers. It is assumed that all flow variables depend on the normal-to-walls coordinate $y$, and are independent of the tangential coordinate $x$. Accordingly, we consider an unsteady one-dimensional and bidirectional (i.e., with nonzero flow velocity components in both $x$ and $y$ directions) problem.

\section{BALLISTIC LIMIT}

In cases where the gap width is small compared with the molecular mean free path, $\mathrm{Kn} \gg 1$, or the excitation timescale is short compared with the mean free time, the effect of molecular collisions is negligible. The gas state is then governed by the collisionless Boltzmann equation,

$$
\frac{\partial f}{\partial t}+\xi_{y} \frac{\partial f}{\partial y}=0
$$

where $f(y, t, \boldsymbol{\xi})$ denotes the probability density function of finding a gas molecule with velocity about $\xi$ at position near $y$ at time $t$. Equation (6) is supplemented by an initial equilibrium condition,

$$
f\left(y, t=0^{-}, \boldsymbol{\xi}\right)=\frac{1}{\pi^{3 / 2}} \exp \left[-\xi^{2}\right],
$$

and fully diffuse boundary conditions,

$$
\begin{aligned}
& f\left(y=-1 / 2, t, \xi_{x}, \xi_{y}>0, \xi_{z}\right)=\frac{\rho_{w_{1}}(t)}{\left[\pi T_{w_{1}}(t)\right]^{3 / 2}} \exp \left[-\frac{\left(\xi_{x}-U_{w_{1}}(t)\right)^{2}+\xi_{y}^{2}+\xi_{z}^{2}}{T_{w_{1}}(t)}\right] \text { and } \\
& f\left(y=1 / 2, t, \xi_{x}, \xi_{y}<0, \xi_{z}\right)=\frac{\rho_{w_{2}}(t)}{\pi^{3 / 2}} \exp \left[-\left(\left(\xi_{x}-U_{w_{2}}(t)\right)^{2}+\xi_{y}^{2}+\xi_{z}^{2}\right)\right] .
\end{aligned}
$$

In Eq. (8), $T_{w_{1}}(t)=1$ in the isothermal wall case, or treated unknown in the adiabatic wall setup [see Eq. (2)]. The problem for $f(y, t, \boldsymbol{\xi})$ is amenable to a general solution for an arbitrary choice of walls tangential velocities $U_{w_{1,2}}(t)$,

$$
f(y, t, \boldsymbol{\xi})= \begin{cases}\frac{\rho_{w_{1}}\left(t_{r_{1}}\right)}{\left[\pi T_{w_{1}}\left(t_{r_{1}}\right)\right]^{3 / 2}} \exp \left[-\frac{\left(\xi_{x}-U_{w_{1}}\left(t_{r_{1}}\right)\right)^{2}+\xi_{y}^{2}+\xi_{z}^{2}}{T_{w_{1}}\left(t_{r_{1}}\right)}\right], & \xi_{y}>(y+1 / 2) / t, \\ \frac{\rho_{w_{2}}\left(t_{r_{2}}\right)}{\pi^{3 / 2}} \exp \left[-\left(\left(\xi_{x}-U_{w_{2}}\left(t_{r_{2}}\right)\right)^{2}+\xi_{y}^{2}+\xi_{z}^{2}\right)\right], & \xi_{y}<(y-1 / 2) / t, \\ \frac{1}{\pi^{3 / 2}} \exp \left[-\left(\xi_{x}^{2}+\xi_{y}^{2}+\xi_{z}^{2}\right)\right], & (y-1 / 2) / t<\xi_{y}<(y+1 / 2) / t,\end{cases}
$$

where $t_{r_{1,2}}=t-(y \pm 1 / 2) / \xi_{y}$ denote the acoustic retarded times corresponding to particle collisions with the $y=\mp 1 / 2$ walls, respectively. The $\xi_{y}$-intervals appearing in Eq. (9) separate between particles which recent wall impact after $t=0$ took place 
with the $y=-1 / 2$ boundary $\left[\xi_{y}>(y+1 / 2) / t\right]$, or $y=+1 / 2$ boundary $\left.\left[\xi_{y}<(y-1 / 2) / t\right)\right]$, or particles which have not collided yet with neither of the surfaces after $t=0\left[(y-1 / 2) / t<\xi_{y}<(y+1 / 2) / t\right]$. With increasing time, the latter interval diminishes, and the medium consists of particles which recent collision at $t>0$ occurred with one of the walls. The functions $\rho_{w_{1,2}}(t)$ and $T_{w_{1}}(t)$ appearing in Eq. (9) are determined via imposition of the macroscopic no-penetration and heat-flux conditions,

$$
\frac{1}{\pi^{3 / 2}} \int_{-\infty}^{\infty} \xi_{y} f(y=\mp 1 / 2) d \xi=0 \quad \text { and } \quad \frac{1}{2 \pi^{3 / 2}} \int_{-\infty}^{\infty} \xi_{y}\left[\left(\xi_{x}-u(-1 / 2, t)\right)^{2}+\xi_{y}^{2}+\xi_{z}^{2}\right] f(y=-1 / 2) d \xi=0,
$$

respectively, where $u(-1 / 2, t)$ is the macroscopic tangential gas velocity at the $y=-1 / 2$ surface. For the case of an isothermal wall at $y=-1 / 2$, the adiabatic condition is omitted $\left(T_{w_{1}}=1\right)$, and only the impermeability conditions are applied to determine $\rho_{w_{1,2}}(t)$. Substituting Eq. (9) into Eq. (10) and assigning the change of variables $\xi_{y}=1 /(t-\tau)$ yield a system of coupled nonlinear integral equations for $\rho_{w_{1,2}}$ and $T_{w_{1}}$,

$$
\begin{gathered}
\rho_{w_{1}}(t) T_{w_{1}}^{1 / 2}(t)=1-\exp \left[-1 / t^{2}\right]+2 \int_{0}^{t} \frac{\rho_{w_{2}}(\tau)}{(t-\tau)^{3}} \exp \left[-\frac{1}{(t-\tau)^{2}}\right] d \tau, \\
\rho_{w_{2}}(t)=1-\exp \left[-1 / t^{2}\right]+2 \int_{0}^{t} \frac{\rho_{w_{1}}(\tau)}{T_{w_{1}}^{1 / 2}(\tau)(t-\tau)^{3}} \exp \left[-\frac{1}{T_{w_{1}}(\tau)(t-\tau)^{2}}\right] d \tau,
\end{gathered}
$$

and

$$
\begin{aligned}
& \rho_{w_{1}}(t) T_{w_{1}}^{1 / 2}(t)\left[\frac{\left(U_{w_{1}}(t)-u(-1 / 2, t)\right)^{2}}{2}+T_{w_{1}}(t)\right]=\left(1+\frac{1}{2} u^{2}(-1 / 2, t)\right)\left[1-\exp \left(-1 / t^{2}\right)\right] \\
& -\frac{1}{2 t^{2}} \exp \left(-1 / t^{2}\right)+\int_{0}^{t} \frac{\rho_{w_{2}}(\tau)}{(t-\tau)^{3}}\left[1+\left[U_{w_{2}}(\tau)-u(-1 / 2, t)\right]^{2}+\frac{1}{(t-\tau)^{2}}\right] \exp \left[-\frac{1}{(t-\tau)^{2}}\right] d \tau,
\end{aligned}
$$

subject to the initial conditions

$$
T_{w_{1}}(0)=\rho_{w_{1}}(0)=\rho_{w_{2}}(0)=1 .
$$

The problem is discretized in time and integrated numerically using the Euler method, starting at $t=0$ with the conditions in Eq. (14).

Once $\rho_{w_{1,2}}$ and $T_{w_{1}}$ are calculated, the solution in Eq. (9) for $f(y, t, \xi)$ is known, and the hydrodynamic fields are obtained via quadratures over the velocity space. Explicit expressions for the density $\rho$, tangential velocity $u$, normal velocity $v$, pressure $p$, and normal heat-flux $q_{y}$ are given in Appendix A. For later reference, we specify expressions for the tangential and normal forces per unit area acting on the $y=-1 / 2$ boundary,

$$
\begin{aligned}
F_{w_{1}}^{(t)}(t)= & \int \xi_{y}\left[\xi_{x}-u(-1 / 2, t)\right] f(y=-1 / 2) d \xi=\frac{1}{\sqrt{\pi}}\left\{\frac{1}{2} \rho_{w_{1}}(t) T_{w_{1}}^{1 / 2}(t)\left[U_{w_{1}}(t)-u(-1 / 2, t)\right]\right. \\
& \left.+\frac{1}{2} u(-1 / 2, t)\left[1-\exp \left(-\frac{1}{t^{2}}\right)\right]-\int_{0}^{t} \rho_{w_{2}}(\tau)\left[U_{w_{2}}(\tau)-u(-1 / 2, t)\right] \exp \left[-\frac{1}{(t-\tau)^{2}}\right] \frac{d \tau}{(t-\tau)^{3}}\right\}
\end{aligned}
$$

and

$$
\begin{aligned}
F_{w_{1}}^{(n)}(t) & =\int \xi_{y}^{2} f(y=-1 / 2) d \xi-\frac{1}{2} \\
& =\frac{\rho_{w_{1}}(t) T_{w_{1}}(t)}{4}+\frac{1}{4} \operatorname{erf}\left(\frac{1}{t}\right)-\frac{1}{2 \sqrt{\pi} t} \exp \left[-\frac{1}{t^{2}}\right]+\frac{1}{\sqrt{\pi}} \int_{0}^{t} \rho_{w_{2}}(\tau) \exp \left[-\frac{1}{(t-\tau)^{2}}\right] \frac{d \tau}{(t-\tau)^{4}}-\frac{1}{2},
\end{aligned}
$$

respectively, where $\operatorname{erf}(\cdot)$ marks the Error Function of a variable. The counterpart forces on the $y=+1 / 2$ boundary are

$$
\begin{aligned}
F_{w_{2}}^{(t)}(t)= & \int \xi_{y}\left(\xi_{x}-u(1 / 2, t)\right) f(y=1 / 2) d \xi=\frac{1}{\sqrt{\pi}}\left\{-\frac{1}{2} \rho_{w_{2}}(t)\left[U_{w_{2}}(t)-u(1 / 2, t)\right]\right. \\
& \left.+\int_{0}^{t} \frac{\rho_{w_{1}}(\tau)}{T_{w_{1}}^{1 / 2}(\tau)}\left[U_{w_{1}}(\tau)-u(1 / 2, t)\right] \exp \left[-\frac{1}{T_{w_{1}}(\tau)(t-\tau)^{2}}\right] \frac{d \tau}{(t-\tau)^{3}}-\frac{1}{2} u(1 / 2, t)\left[1-\exp \left(-1 / t^{2}\right)\right]\right\}
\end{aligned}
$$

and

$$
\begin{aligned}
F_{w_{2}}^{(n)}(t)= & \int \xi_{y}^{2} f(\mathrm{y}=1 / 2) \mathrm{d} \xi-\frac{1}{2}=\frac{\rho_{w_{2}}(t)}{4}+\frac{1}{4} \operatorname{erf}\left(\frac{1}{t}\right)-\frac{1}{2 \sqrt{\pi} t} \exp \left(-\frac{1}{t^{2}}\right) \\
& +\frac{1}{\sqrt{\pi}} \int_{0}^{t} \frac{\rho_{w_{1}}(\tau)}{T_{w_{1}}^{1 / 2}(\tau)} \exp \left[-\frac{1}{T_{w_{1}}(\tau)(t-\tau)^{2}}\right] \frac{d \tau}{(t-\tau)^{4}}-\frac{1}{2} .
\end{aligned}
$$


In Eqs. (16) and (18), the normal force $F_{\mathrm{eq}}^{(n)}=1 / 2$ acting in equilibrium has been subtracted, so that $F_{w_{1}}^{(n)}(t)$ and $F_{w_{2}}^{(n)}(t)$ measure only the deviations from equilibrium of the normal forces on the walls. Thus, it can be verified that at isothermal conditions, where $T_{w_{1}}(t)=\rho_{w_{1}}(t)=\rho_{w_{2}}(t)=1$, both $F_{w_{1}}^{(n)}(t)$ and $F_{w_{2}}^{(n)}(t)$ vanish.

\section{CONTINUUM LIMIT}

When the slab width is large compared with the molecular mean free path, $\mathrm{Kn} \ll 1$, and the excitation timescale is long compared with the mean free time, the effect of molecular collisions becomes dominant, and continuum-limit conditions prevail. We then make use of a 'slip-flow' description, combining the Navier-Stokes-Fourier equations with first-order velocity slip and temperature jump boundary conditions. Assuming a one-dimensional bidirectional flow setup and adopting the scaling introduced in Sec. II, the continuity, $x$ momentum, $y$ momentum, and energy equations are given by

$$
\begin{gathered}
\frac{\partial \rho}{\partial t}+\frac{\partial(\rho v)}{\partial y}=0, \\
\rho\left(\frac{\partial u}{\partial t}+v \frac{\partial u}{\partial y}\right)=\widetilde{\mathrm{Kn}} \frac{\partial}{\partial y}\left(\mu \frac{\partial u}{\partial y}\right), \\
\rho\left(\frac{\partial v}{\partial t}+v \frac{\partial v}{\partial y}\right)=-\frac{\partial p}{\partial y}+\frac{4}{3} \widetilde{\mathrm{Kn}} \frac{\partial}{\partial y}\left(\mu \frac{\partial v}{\partial y}\right),
\end{gathered}
$$

and

$$
\begin{aligned}
\rho\left(\frac{\partial T}{\partial t}+v \frac{\partial T}{\partial y}\right)= & \frac{\gamma \widetilde{\mathrm{Kn}}}{\operatorname{Pr}} \frac{\partial}{\partial y}\left(\kappa \frac{\partial T}{\partial y}\right)-(\gamma-1) p \frac{\partial v}{\partial y} \\
& +2 \widetilde{\mathrm{Kn}}(\gamma-1) \mu\left[\left(\frac{\partial u}{\partial y}\right)^{2}+\frac{4}{3}\left(\frac{\partial v}{\partial y}\right)^{2}\right]
\end{aligned}
$$

respectively, and are supplemented by the equation of state for an ideal gas,

$$
p=\frac{1}{2} \rho T
$$

In Eqs. (20)-(22),

$$
\widetilde{\mathrm{Kn}}=\frac{\mu_{0}^{*}}{\rho_{0}^{*} U_{\mathrm{th}}^{*} L^{*}}
$$

is the viscosity-based Knudsen number, where $\mu_{0}^{*}$ marks the transport coefficient of dynamic viscosity at $T^{*}=T_{0}^{*}$. In addition, $\operatorname{Pr}$ and $\gamma$ denote the Prandtl number and ratio of specific heats at constant pressure and volume, respectively, and $\mu$ and $\kappa$ are the nondimensional coefficients of dynamic viscosity and heat conduction, respectively, scaled by their reference values at $T^{*}=T_{0}^{*}$. Considering a monatomic hard-sphere gas, $\operatorname{Pr}=2 / 3$ and $\gamma=5 / 3, \mu(T)=\kappa(T)=T^{1 / 2}$, and $\widetilde{\mathrm{Kn}}=$ $(5 \sqrt{\pi} / 16) \mathrm{Kn}$ [32]. The problem is complemented by the velocity-slip and impermeability conditions at the walls,

$v(\mp 1 / 2, t)=0$ and $u(\mp 1 / 2, t)=U_{w_{1,2}}(t) \pm\left.\zeta \frac{\partial u}{\partial y}\right|_{(\mp 1 / 2, t)}$, together with a temperature jump condition at $y=1 / 2$,

$$
T(1 / 2, t)=1-\left.\vartheta_{T} \frac{\partial T}{\partial y}\right|_{(1 / 2, t)}+\left.\vartheta_{v} \frac{\partial v}{\partial y}\right|_{(1 / 2, t)},
$$

and an adiabatic or temperature jump condition at $y=-1 / 2$,

$$
\begin{aligned}
& q_{y}(-1 / 2, t)=-\left.\kappa \frac{\partial T}{\partial y}\right|_{(-1 / 2, t)}=0 \quad \text { or } \\
& T(-1 / 2, t)=1+\left.\vartheta_{T} \frac{\partial T}{\partial y}\right|_{(-1 / 2, t)}+\left.\vartheta_{v} \frac{\partial v}{\partial y}\right|_{(-1 / 2, t)},
\end{aligned}
$$

respectively. In Eqs. (25)-(27), $\zeta=1.254 \sqrt{\pi} \mathrm{Kn} / 2$ and $\vartheta_{T}=$ $2.399 \sqrt{\pi} \mathrm{Kn} / 2$ are the commonly used first-order nondimensional slip and jump coefficients for a hard-sphere gas, respectively [32]. The $\vartheta_{v}(\partial v / \partial y)$ terms with $\vartheta_{v}=0.460 \sqrt{\pi} \mathrm{Kn} / 2$ mark an additional jump correction, recently derived by Aoki et al. [33], to accompany the compressible Navier-Stokes equations.

Different from the ballistic-flow formulation, the continuum-limit problem is not amenable to a closed-form solution for arbitrary walls excitation. We therefore limit our discussion to harmonic boundaries oscillations as specified in Eq. (4), and seek for a Mach number expansion of the solution, to study the leading-order impact of nonsmall wall velocities on the gas deviation from its isothermal state. Inspection of Eqs. (19)-(23) and (25)-(27) suggests the following Mach power representation of the hydrodynamic fields,

$$
\begin{aligned}
u & =\operatorname{Ma} u^{(1)}(y, t)+O\left(\mathrm{Ma}^{3}\right), v=\mathrm{Ma}^{2} v^{(2)}(y, t)+O\left(\mathrm{Ma}^{4}\right), \\
\rho & =1+\mathrm{Ma}^{2} \rho^{(2)}(y, t)+O\left(\mathrm{Ma}^{4}\right), \\
T & =1+\mathrm{Ma}^{2} T^{(2)}(y, t)+O\left(\mathrm{Ma}^{4}\right) \text { and } \\
p & =\frac{1}{2}+\mathrm{Ma}^{2} p^{(2)}(y, t)+O\left(\mathrm{Ma}^{4}\right),
\end{aligned}
$$

to be valid at relatively small Mach numbers. In the following we apply the above expansion to calculate the system behavior up to $O\left(\mathrm{Ma}^{2}\right)$.

Substituting Eq. (28) into Eqs. (19)-(23) and (25)-(27) together with Eq. (4), the $O(\mathrm{Ma})$ problem contains the leading order $x$-momentum equation,

$$
\frac{\partial u^{(1)}}{\partial t}=\widetilde{\mathrm{Kn}} \frac{\partial^{2} u^{(1)}}{\partial y^{2}},
$$

together with the velocity-slip conditions

$$
\begin{gathered}
u^{(1)}(-1 / 2, t)=\cos (\omega t)+\left.\zeta \frac{\partial u^{(1)}}{\partial y}\right|_{(-1 / 2, t)}, \\
u^{(1)}(1 / 2, t)=-\cos (\omega t)-\left.\zeta \frac{\partial u^{(1)}}{\partial y}\right|_{(1 / 2, t)} .
\end{gathered}
$$

Assuming harmonic time dependence of the solution,

$$
u^{(1)}(y, t)=\operatorname{Re}\left\{\bar{u}^{(1)}(y) \exp [\mathrm{i} \omega t]\right\},
$$

and substituting into Eq. (29), yields

$$
\bar{u}^{(1)}(y)=A_{1} \exp \left[\sqrt{\frac{\mathrm{i} \omega}{\widetilde{\mathrm{Kn}}}} y\right]+A_{2} \exp \left[-\sqrt{\frac{\mathrm{i} \omega}{\widetilde{\mathrm{Kn}}}} y\right],
$$


where the complex constants of integration $A_{1,2} \equiv r_{1,2} e^{\mathrm{i} \theta_{1,2}}$ are fixed through the boundary conditions (30), and are not specified here for brevity. Expectedly, the leading $O(\mathrm{Ma})$ solution captures the system isothermal state, where all thermodynamic variables remain unperturbed [15]. The coupling between the gas dynamic and thermodynamic descriptions is therefore manifested via the next $O\left(\mathrm{Ma}^{2}\right)$ balance, which becomes significant at nonsmall Mach numbers. Eq. (32) also indicates an exponential $\exp \left[(\omega / \widetilde{\mathrm{Kn}})^{1 / 2}\right]$ dependence of the solution. To comply with the near-continuum conditions, it is therefore required, apart from assuming $\mathrm{Kn} \sim \widetilde{\mathrm{Kn}} \ll 1$, that $\omega \lesssim \mathrm{Kn}$. This requirement prevents confinement of the flow-field to thin layers in the vicinity of the boundaries, in which case the effective Knudsen number increases, and the continuum description breaks down.

Proceeding to the $O\left(\mathrm{Ma}^{2}\right)$ balance, substitution of Eq. (28) into Eqs. (19)-(23) and (25)-(27) yields the coupled $O\left(\mathrm{Ma}^{2}\right)$ balances of continuity,

$$
\frac{\partial \rho^{(2)}}{\partial t}+\frac{\partial v^{(2)}}{\partial y}=0
$$

$y$ momentum,

$$
\frac{\partial v^{(2)}}{\partial t}=-\frac{1}{2}\left(\frac{\partial \rho^{(2)}}{\partial y}+\frac{\partial T^{(2)}}{\partial y}\right)+\frac{4}{3} \widetilde{\mathrm{Kn}} \frac{\partial^{2} v^{(2)}}{\partial y^{2}},
$$

and energy,

$$
\begin{aligned}
\frac{\partial T^{(2)}}{\partial t}= & \frac{\gamma \widetilde{\mathrm{Kn}}}{\operatorname{Pr}} \frac{\partial^{2} T^{(2)}}{\partial y^{2}}-(\gamma-1) \frac{\partial v^{(2)}}{\partial y} \\
& +2(\gamma-1) \widetilde{\mathrm{Kn}}\left(\frac{\partial u^{(1)}}{\partial y}\right)^{2}
\end{aligned}
$$

equations, supplemented by the impermeability condition at both walls,

$$
v^{(2)}(\mp 1 / 2, t)=0,
$$

temperature jump condition at $y=1 / 2$,

$$
T^{(2)}(+1 / 2, t)=-\left.\vartheta_{T} \frac{\partial T^{(2)}}{\partial y}\right|_{(1 / 2, t)}+\left.\vartheta_{v} \frac{\partial v^{(2)}}{\partial y}\right|_{(1 / 2, t)}
$$

and adiabatic or temperature jump condition at $y=-1 / 2$,

$$
\begin{aligned}
& \left.\frac{\partial T^{(2)}}{\partial y}\right|_{(-1 / 2, t)}=0 \text { or } \\
& T^{(2)}(-1 / 2, t)=\left.\vartheta_{T} \frac{\partial T^{(2)}}{\partial y}\right|_{(-1 / 2, t)}+\left.\vartheta_{v} \frac{\partial v^{(2)}}{\partial y}\right|_{(-1 / 2, t)} .
\end{aligned}
$$

A nontrivial solution to the above problem is attributed to the dissipation term in Eq. (35), coupling the square of the leading order tangential velocity gradient $\left(\partial u^{(1)} / \partial y\right)^{2}$ as a forcing term. It is therefore due to the effect of viscous dissipation that the thermodynamic and dynamic gas states are coupled in the continuum limit.

Inspecting the quadratic form of the forcing term, and in view of the $\omega$-harmonic time dependence of the solution in Eq. (31) for $u^{(1)}$, we decompose the solution for the $O\left(\mathrm{Ma}^{2}\right)$ fields into steady and unsteady double-harmonic components,

$$
\begin{aligned}
& {\left[v^{(2)}, \rho^{(2)}, T^{(2)}\right](y, t)=\left[v_{s}^{(2)}, \rho_{s}^{(2)}, T_{s}^{(2)}\right](y)} \\
& \quad+\operatorname{Re}\left\{\left[\bar{v}^{(2)}, \bar{\rho}^{(2)}, \bar{T}^{(2)}\right](y) \exp [2 \mathrm{i} \omega t]\right\} .
\end{aligned}
$$

Assigning Eq. (39) into Eqs. (33)-(35) yields separate sets of equations for the steady,

$$
\begin{aligned}
& \frac{d v_{s}^{(2)}}{d y}=0, \frac{d p_{s}^{(2)}}{d y}=0, \frac{d^{2} T_{s}^{(2)}}{d y^{2}}=-\frac{2 \operatorname{Pr}(\gamma-1)}{\gamma} F_{s}^{(2)}, \\
& p_{s}^{(2)}=\frac{1}{2}\left(\rho_{s}^{(2)}+T_{s}^{(2)}\right),
\end{aligned}
$$

and time-periodic,

$$
\begin{aligned}
& 2 \mathrm{i} \omega \bar{\rho}^{(2)}+\frac{d \bar{v}^{(2)}}{d y}=0,2 \mathrm{i} \omega \bar{v}^{(2)}=-\frac{1}{2} \frac{d \bar{p}^{(2)}}{d y}+\frac{4}{3} \widetilde{\mathrm{Kn}} \frac{d^{2} \bar{v}^{(2)}}{d y^{2}}, \\
& 2 \mathrm{i} \omega \bar{T}^{(2)}=\frac{\gamma \widetilde{\mathrm{Kn}}}{\operatorname{Pr}} \frac{d^{2} \bar{T}^{(2)}}{d y^{2}}-(\gamma-1) \frac{d \bar{v}^{(2)}}{d y}+2(\gamma-1) \widetilde{\mathrm{Kn}} \bar{F}^{(2)}, \\
& \bar{p}^{(2)}=\frac{1}{2}\left(\bar{\rho}^{(2)}+\bar{T}^{(2)}\right),
\end{aligned}
$$

parts, where $F_{s}^{(2)}=\left|d \bar{u}^{(1)} / d y\right|^{2} / 2$ and $\bar{F}^{(2)}=\left(d \bar{u}^{(1)} / d y\right)^{2} / 2$ are the steady and $2 \omega$-oscillatory components of $\left(\partial u^{(1)} / \partial y\right)^{2}$, respectively. Starting with the problem for the steady part, Eq. (40) yields the general solution,

$v_{s}^{(2)}=0, \quad T_{s}^{(2)}=-\frac{2 \operatorname{Pr}(\gamma-1)}{\gamma} \iint F_{s}^{(2)} d^{2} y+D_{1} y+D_{2}$,

where the constant $p_{s}^{(2)}$ is determined via the normalization condition,

$$
\int_{-1 / 2}^{1 / 2} \rho_{s}^{(2)} d y=0
$$

imposing the conservation of gas mass within the slab. The constants $D_{1,2}$ in Eq. (42) are fixed through the thermal wall conditions,

$$
T_{s}^{(2)}(1 / 2)=-\left.\vartheta_{T} \frac{d T_{s}^{(2)}}{d y}\right|_{1 / 2}
$$

and

$$
\left.\frac{d T_{s}^{(2)}}{d y}\right|_{-1 / 2}=0 \quad \text { or } \quad T_{s}^{(2)}(-1 / 2)=\left.\vartheta_{T} \frac{d T_{s}^{(2)}}{d y}\right|_{-1 / 2} .
$$

Explicit calculation yields

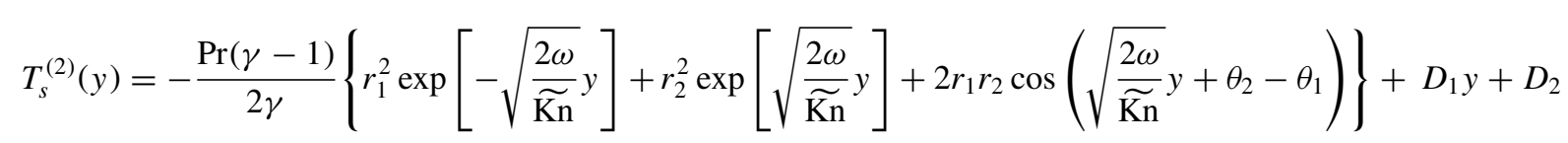


and

$$
p_{s}^{(2)}=-\frac{\operatorname{Pr}(\gamma-1)}{2 \gamma} \sqrt{\frac{\widetilde{\mathrm{Kn}}}{2 \omega}}\left\{\left(r_{2}^{2}-r_{1}^{2}\right) \sinh \left[\sqrt{\frac{\omega}{2 \widetilde{\mathrm{Kn}}}}\right]+2 r_{1} r_{2} \sin \left(\sqrt{\frac{\omega}{2 \widetilde{\mathrm{Kn}}}}\right) \cos \left(\theta_{2}-\theta_{1}\right)\right\}+\frac{1}{2} D_{2},
$$

where $r_{1,2}$ and $\theta_{1,2}$ denote the magnitudes and arguments of the complex coefficients $A_{1,2}$, introduced after Eq. (32).

Turning to the periodic component, the system of Eqs. (41) may be reduced to a single nonhomogeneous equation for the temperature,

$k m \frac{d^{4} \bar{T}^{(2)}}{d y^{4}}-\left[\frac{1}{2}(\gamma-1)+2 \mathrm{i} \omega(k+m)\right] \frac{d^{2} \bar{T}^{(2)}}{d y^{2}}-4 \omega^{2} \bar{T}^{(2)}=2(\gamma-1) \widetilde{\mathrm{Kn}}\left\{\mathrm{i} \omega\left(\frac{d \bar{u}^{(1)}}{d y}\right)^{2}-m\left[\left(\frac{d^{2} \bar{u}^{(1)}}{d y^{2}}\right)^{2}+\frac{d \bar{u}^{(1)}}{d y} \frac{d^{3} \bar{u}^{(1)}}{d y^{3}}\right]\right\}$,

where $k=\gamma \widetilde{\mathrm{Kn}} / \operatorname{Pr}$ and $m=4 \widetilde{\mathrm{Kn}} / 3-\mathrm{i} / 4 \omega$. Expressing $\bar{T}^{(2)}(y)$ as a sum of a homogeneous and particular solutions,

$$
\bar{T}^{(2)}(y)=\bar{T}_{h}^{(2)}(y)+\bar{T}_{p}^{(2)}(y),
$$

respectively, we find

$$
\begin{array}{r}
\bar{T}_{h}^{(2)}(y)=B_{1} \exp \left[-\sqrt{\lambda_{1}} y\right]+B_{2} \exp \left[-\sqrt{\lambda_{2}} y\right]+B_{3} \exp \left[\sqrt{\lambda_{1}} y\right]+B_{4} \exp \left[\sqrt{\lambda_{2}} y\right] \\
\text { and } \bar{T}_{p}^{(2)}(y)=C_{1} \exp \left[-2 \sqrt{\frac{\mathrm{i} \omega}{\widetilde{\mathrm{Kn}}}} y\right]+C_{2} \exp \left[2 \sqrt{\left.\frac{\mathrm{i} \omega}{\widetilde{\mathrm{Kn}}} y\right]+C_{3},}\right.
\end{array}
$$

where $\lambda_{1,2}$ are the roots of the characteristic equation

$$
k m \lambda^{2}-\left[\frac{1}{2}(\gamma-1)+2 \mathrm{i} \omega(k+m)\right] \lambda-4 \omega^{2}=0 .
$$

Substituting the particular solution $\bar{T}_{p}^{(2)}(y)$ in Eq. (50) into Eq. (48) yields

$$
C_{1,2}=\frac{\operatorname{Pr}(\gamma-1)(3 \mathrm{i}-10 \widetilde{\mathrm{Kn}} \omega)}{6 \mathrm{i} \gamma(\operatorname{Pr}-2)+20 \widetilde{\mathrm{Kn}} \omega(2 \gamma-\operatorname{Pr})} A_{1,2}^{2} \text { and } C_{3}=-(\gamma-1) A_{1} A_{2} .
$$

The coefficients $B_{1,2,3,4}$ in $\bar{T}_{h}^{(2)}(y)$ are obtained via application of the impermeability and thermal boundary conditions to Eq. (49) together with Eqs. (50) and (52). Having specified $\bar{T}^{(2)}(y)$, the periodic-component amplitude functions of the normal velocity $\bar{v}^{(2)}(y)$ and density $\bar{\rho}^{(2)}(y)$ are found via substitutions into Eq. (41), yielding the expressions presented in Appendix B.

Summing the $O(\mathrm{Ma})$ and $O\left(\mathrm{Ma}^{2}\right)$ contributions, the total forces per unit area acting on the $y=\mp 1 / 2$ boundaries in the tangential and normal directions (subtracting the normal equilibrium force $F_{\mathrm{eq}}^{(n)}=1 / 2$; cf. Eqs. (15)-(18) et seq.) are

$$
F_{w_{1,2}}^{(t)}(t)=\operatorname{Re}\left\{\operatorname{Ma} \widetilde{K n} \frac{d \bar{u}^{(1)}}{d y}(\mp 1 / 2, t) \exp [\mathrm{i} \omega t]\right\}
$$

and

$$
F_{w_{1,2}}^{(n)}(t)=-\operatorname{Ma}^{2} p_{s}^{(2)}+\operatorname{Re}\left\{\operatorname{Ma}^{2}\left[-\bar{p}^{(2)}(\mp 1 / 2, t)+\frac{4}{3} \widetilde{\mathrm{Kn}} \frac{d \bar{v}^{(2)}}{d y}(\mp 1 / 2, t)\right] \exp [2 \mathrm{i} \omega t]\right\},
$$

respectively. Notably, while the $O(\mathrm{Ma})$ tangential force is $\omega$ periodic, the $O\left(\mathrm{Ma}^{2}\right)$ normal component contains both steady and unsteady $2 \omega$-periodic contributions. Being a consequence of the form of solution in Eq. (39), this result will be further validated and discussed in Sec. VI.

\section{NUMERICAL SCHEME: DSMC METHOD}

The direct simulation Monte Carlo method proposed by Bird [34] is a stochastic particle method commonly applied for the analysis of rarefied gas flows. In the present work, we apply the DSMC method to validate the analytical ballisticand continuum-limit solutions presented in Secs. III and IV.
We adopt Bird's algorithm and assume hard-sphere interactions between the gas molecules. In line with problem formulation, the boundaries are assumed fully diffuse, with prescribed heat-flux or temperature. In the former case, and in difference from traditional applications of the wall-interaction algorithm, the boundary temperature is treated as unknown. Realization of a heat-flux condition therefore requires modification of the conventional calculation.

In a recent contribution by the authors [35], a noniterative procedure for the imposition of a heat-flux condition in a DSMC calculation has been suggested. The algorithm was assigned in a pulsating cylinder setup, where the gas tangential velocity is assumed to vanish throughout the flow domain. The present shear-flow configuration is qualitatively 
different due to the occurrence of gas tangential velocity. It therefore requires further adjustments that take account of the tangential slip-flow effects. In what follows we describe the algorithm for wall heat-flux imposition in DSMC in the presence of gas tangential velocity. For generality, we consider a wall with arbitrarily prescribed (dimensional) normal heat-flux $Q_{w}^{*}\left(t^{*}\right)$. For an adiabatic wall, $Q_{w}^{*}\left(t^{*}\right)=0$ should be inserted.

Consider the interaction of gas molecules with a diffuse reflecting boundary displaced at tangential velocity $U_{w}^{*}\left(t^{*}\right)$. The distribution of the velocity components for the wallreflected molecules in the $i$ th time step is

$$
u^{* i}=\frac{1}{\sqrt{2} \beta_{w}^{*^{i}}} R_{G}+U_{w}^{*^{i}}, \quad v^{*^{i}}=\frac{1}{\beta_{w}^{*^{i}}} \sqrt{-\ln \left(R_{U}\right)}, \quad \text { and } \quad w^{*^{i}}=\frac{1}{\sqrt{2} \beta_{w}^{*^{i}}} R_{G},
$$

in the tangential $x^{*}$, normal $y^{*}$, and normal-to-slab $z^{*}$ directions, respectively. In Eq. (55), $R_{U} \in[0,1]$ and $R_{G} \in(-\infty, \infty)$ are uniformly distributed and Gaussian-distributed random numbers, and $\beta_{w}^{*^{i}}=\sqrt{1 / 2 \mathcal{R}^{*} T_{w}^{*^{i}}}$. Assigning a heat-flux condition, the value of $\beta_{w}^{*^{i}}$ is apriori unknown at every time step and should be determined. For the fully diffuse boundary considered here, the total mass and thermal energy fluxes of the reflected molecules are

$$
\mathcal{M}_{\text {out }}^{*^{i}}=\frac{\rho_{w}^{*^{i}}\left(\beta_{w}^{*^{i}}\right)^{3}}{\pi^{3 / 2}} \int_{\xi_{y}^{*>0}} \xi_{y}^{*} \exp \left[-\left(\beta_{w}^{*^{i}}\right)^{2}\left(\left(\xi_{x}^{*}-U_{w}^{*^{i}}\right)^{2}+\xi_{y}^{*^{2}}+\xi_{z}^{*^{2}}\right)\right] d \xi^{*}=\frac{\rho_{w}^{*^{i}}}{2 \sqrt{\pi} \beta_{w}^{*^{i}}}
$$

and

$$
\begin{aligned}
\mathcal{H}_{\mathrm{out}}^{*^{i}} & =\frac{\rho_{w}^{*^{i}}\left(\beta_{w}^{*^{i}}\right)^{3}}{2 \pi^{3 / 2}} \int_{\xi_{y}^{*}>0} \xi_{y}^{*}\left(\left(\xi_{x}^{*}-u_{g}^{*^{i}}\right)^{2}+\xi_{y}^{*^{2}}+\xi_{z}^{*^{2}}\right) \exp \left\{-\left(\beta_{w}^{*^{i}}\right)^{2}\left[\left(\xi_{x}^{*}-U_{w}^{*^{i}}\right)^{2}+\xi_{y}^{*^{2}}+\xi_{z}^{*^{2}}\right]\right\} d \xi^{*} \\
& =\frac{\rho_{w}^{*^{i}}}{2 \sqrt{\pi} \beta_{w}^{*^{i}}}\left[\frac{\left(U_{w}^{*^{i}}-u_{g}^{*^{i}}\right)^{2}}{2}+\frac{1}{\left(\beta_{w}^{*^{i}}\right)^{2}}\right]
\end{aligned}
$$

respectively. In Eq. (57), $u_{g}^{*^{i}}$ marks the macroscopic gas velocity at the wall at the $i$ th timestep, given by the average of tangential velocities of the incoming and reflected gas molecules,

$$
u_{g}^{*^{i}}=\frac{1}{2}\left(u_{g, \text { in }}^{*^{i}}+u_{g, \text { out }}^{*^{i}}\right) .
$$

For the fully diffuse boundary considered here, the macroscopic gas velocity of the reflected molecules is

$$
u_{g, \text { out }}^{*^{i}}=\frac{\int_{\xi_{y}^{*}>0} \xi_{x}^{*} \xi_{y}^{*} \exp \left\{-\left(\beta_{w}^{*^{i}}\right)^{2}\left[\left(\xi_{x}^{*}-U_{w}^{*^{i}}\right)^{2}+\xi_{y}^{*^{2}}+\xi_{z}^{*^{2}}\right]\right\} d \xi^{*}}{\int_{\xi_{y}^{*}>0} \xi_{y}^{*} \exp \left\{-\left(\beta_{w}^{*^{i}}\right)^{2}\left[\left(\xi_{x}^{*}-U_{w}^{*^{i}}\right)^{2}+\xi_{y}^{*^{2}}+\xi_{z}^{*^{2}}\right]\right\} d \xi^{*}}=U_{w}^{*^{i}},
$$

and the macroscopic gas velocity of the approaching molecules is given by

$$
u_{g, \text { in }}^{*^{i}}=\frac{1}{N} \sum_{j=1}^{N} \xi_{x}^{*^{i, j}} .
$$

The summation in Eq. (60) is carried out over all $j=1, \ldots, N$ particles that have collided with the boundary during the $\Delta t^{*}=t^{*^{i}}-t^{*^{i-1}}$ time interval.

Combining Eqs. (56)-(59), we find

$$
\frac{1}{\left(\beta_{w}^{*^{i}}\right)^{2}}=\frac{\mathcal{H}_{\mathrm{out}}^{*^{i}}}{\mathcal{M}_{\mathrm{out}}^{*^{i}}}-\frac{\left(U_{w}^{*^{i}}-u_{g, \text { in }}^{*^{i}}\right)^{2}}{8}
$$

Applying the balances of mass, $\mathcal{M}_{\mathrm{out}}^{*^{i}}=\mathcal{M}_{\mathrm{in}}^{*^{i}}$, and thermal energy, $\mathcal{H}_{\mathrm{out}}^{*^{i}}=\mathcal{H}_{\mathrm{in}}^{*^{i}}+Q_{w}^{*^{i}}$, at the boundary, yields

$$
\frac{1}{\beta_{w}^{*^{i}}}=\sqrt{\frac{\mathcal{H}_{\mathrm{in}}^{*^{i}}+Q_{w}^{*^{i}}}{\mathcal{M}_{\mathrm{in}}^{*^{i}}}-\frac{\left(U_{w}^{*^{i}}-u_{g, \text { in }}^{*^{i}}\right)^{2}}{8}},
$$

where $\mathcal{M}_{\text {in }}^{*^{i}}$ and $\mathcal{H}_{\text {in }}^{*^{i}}$ are computed via

$$
\mathcal{M}_{\mathrm{in}}^{*^{i}}=\frac{N}{\Delta t^{*}} \text { and } \mathcal{H}_{\mathrm{in}}^{*^{i}}=\frac{1}{\Delta t^{*}} \sum_{j=1}^{N} \frac{1}{2}\left[\left(\xi_{x}^{*^{i, j}}-\frac{1}{2} U_{w}^{*^{i}}-\frac{1}{2} u_{g, \text { in }}^{*^{i}}\right)^{2}+\left(\xi_{y}^{*^{i, j}}\right)^{2}+\left(\xi_{z}^{*^{i, j}}\right)^{2}\right] .
$$

Having determined $u_{g, \text { in }}^{*^{i}}, \mathcal{M}_{\mathrm{in}}^{*^{i}}$, and $\mathcal{H}_{\mathrm{in}}^{*^{i}}, \beta_{w}^{*^{i}}$ is obtained using Eq. (62). Each of the reflected particles is then assigned a velocity according to Eq. (55), and the simulation is followed to the next $i+1$ timestep. 


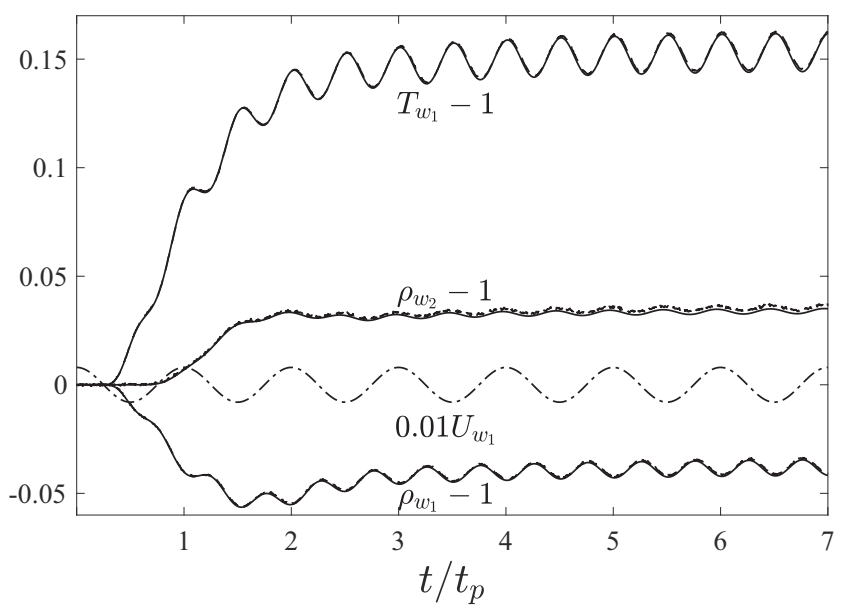

FIG. 1. Time variations (in period $t_{p}=2 \pi / \omega$ units) of the deviations of $T_{w_{1}}, \rho_{w_{1}}$, and $\rho_{w_{2}}$ from their equilibrium initial values for an adiabatic wall setup with $\mathrm{Ma}=0.8$ and $\omega=5$. The solid and dashed curves present the ballistic limit $(\mathrm{Kn} \rightarrow \infty)$ and DSMC $\mathrm{Kn}=72$ results, respectively. The dash-dotted line shows the tangential wall velocity at $y=-1 / 2$, multiplied by 0.01 for scaling.

\section{RESULTS}

Starting with the ballistic flow regime, Fig. 1 presents the deviations of the boundaries-related functions $T_{w_{1}}$ (the $y=-1 / 2$ wall temperature) and $\rho_{w_{1,2}}$ [see Eq. (8)] from their equilibrium initial values. The case of an adiabatic wall setup (where the $y=-1 / 2$ surface is adiabatic) is considered. The solid and dashed curves compare between the ballistic limit $(\mathrm{Kn} \rightarrow \infty)$ and DSMC $\mathrm{Kn}=72$ results, respectively, for $\mathrm{Ma}=0.8$ and $\omega=5$. The time evolutions of $T_{w_{1}}-1$ and $\rho_{w_{1,2}}-1$ are presented, starting at $t=0$ (when boundaries motions commence) through $t=7 t_{p}$, where $t_{p}=2 \pi / \omega$ denotes the period of surfaces oscillations. At that time, the system has reached its final periodic state. Close agreement is obtained between the ballistic analysis and DSMC predictions, and any differences are nearly indiscernible.

Focusing on the early time response, we observe an initial time delay in all fields before system deviation from its thermal equilibrium begins. Specifically, an approximately twice longer time delay is obtained for $\rho_{w_{2}}$ compared with $T_{w_{1}}$ and $\rho_{w_{1}}$. This may be rationalized by means of system kinematics. At $t=0$, the gas is set in uniform equilibrium [see Eq. (9)]. Initial increase in $T_{w_{1}}$ then takes place only after molecules that have collided with the $y=1 / 2$ boundary at $t \geqslant 0$ (and acquired tangential momentum from the oscillating surface) collide with the adiabatic wall. This rise in $T_{w_{1}}$, accompanied by a decrease in $\rho_{w_{1}}$, causes larger normal velocity of the molecules departing from the adiabatic wall. Once these molecules impact the isothermal wall, a consequent increase in $\rho_{w_{2}}$ occurs. The characteristic response time of the isothermal wall is therefore twice the delay time of the adiabatic boundary, as supported by Fig. 1.

Inspecting the late time response in Fig. 1, we observe that $\rho_{w_{1,2}}$ and $T_{w_{1}}$ acquire final states characterized by nonzero mean values, superposed by an essentially $2 \omega$-frequency (compared with the $\omega$-frequency dependence of $U_{w_{1}}$; cf. the dash-dotted line) periodic signal. The nonzero mean values are attributed to the increase in gas kinetic energy, proportional to $u^{2}(t)$, caused by the walls motion, and leading to an increase in the adiabatic wall temperature $T_{w_{1}}$. As observed in Eq. (13), it is the quadratic dependence on the tangential velocity appearing in the adiabatic-wall condition that causes the double-frequency (and higher even harmonics of $\omega$; see the discussion in Fig. 7) dependence of the solution. This is reminiscent of the result obtained in the continuum limit [see Eq. (35) et seq.], showing the leading $O\left(\mathrm{Ma}^{2}\right)$ double-frequency time dependence of the thermal flow variables and normal velocity. In view of the similar behaviors in the ballistic and continuum limits, it is expected that the double-frequency time dependence should dominate the system thermal response at all rarefaction conditions. This is indeed supported by our DSMC calculations below (see Fig. 7). A similar trend was also observed in the numerical results of Refs. [19] and [21].

Having described the initial and transient system responses at $\mathrm{Kn} \gg 1$, the remaining of this section considers the system periodic behavior obtained at long times. Toward this end, Fig. 2 shows snapshots at period time of the tangential velocity $u$, the deviations of the density $\rho$ and temperature $T$ from their unity equilibrium values, and the normal heat flux $q_{y}$, for $\mathrm{Ma}=0.8$ and $\omega=5$. Ballistic flow results are compared with DSMC $\mathrm{Kn}=72$ calculations, for both adiabatic (adiabatic $y=-1 / 2$ surface) and isothermal (isothermal $y=-1 / 2$ surface) wall setups. The agreement between the analytical and DSMC predictions is gratifying, supporting the accurateness of the nonlinear ballistic analysis. Any small discrepancies [visible particularly in the low-amplitude density signal in Fig. 2(b)] may be attributed to the noninfinite Knudsen number used in DSMC computations, and can be further reduced by increasing the simulated $\mathrm{Kn}$.

Inspecting the ballistic-flow isothermal wall results, we recall that $T_{w_{1}}=1$ in this case. Hence, Eq. (13) is omitted from the analysis, and Eqs. (11) and (12) yield $\rho_{w_{1,2}}(t)=1$. At these conditions, it may be readily verified that $\rho(y, t)-$ $1=v(y, t)=0$ [see Eqs. (A1) and (A3), supported by Figs. 2(b) and 4(a)], and only the temperature, pressure and heat flux fields deviate from their equilibrium distributions. The isothermal wall configuration exhibits symmetry properties about $y=0$, yielding symmetric temperature field and antisymmetric tangential velocity and heat flux. No such symmetry exists in the adiabatic wall setup, where elevated temperatures and normal heat fluxes are observed due to the increase in the $y=-1 / 2$ insulated wall temperature (see Fig. 1). Notably, the tangential flow velocity remains nearly unaffected by the change in the walls thermal conditions for the present choice of the Mach number.

Turning to the continuum limit of small $\mathrm{Kn}$ and $\omega$, Figure 3 presents counterpart results for the tangential velocity, density and temperature deviations, and normal heat flux, for $\mathrm{Ma}=$ $0.3, \omega=0.1$, and $\mathrm{Kn}=0.09$. Adiabatic and isothermal walls setup are presented and compared between slip-flow analysis and DSMC computations at period time. The symmetry properties of the isothermal-setup fields are clearly observed, in contrast with the adiabatic-wall configuration. The DSMC results in Fig. 3(a) confirm that nonlinear thermal effects [being $O\left(\mathrm{Ma}^{3}\right)$ in accordance with the Mach power expansion in Eq. (28)], at the $\mathrm{Ma}=0.3$ value presented, have nearly no 

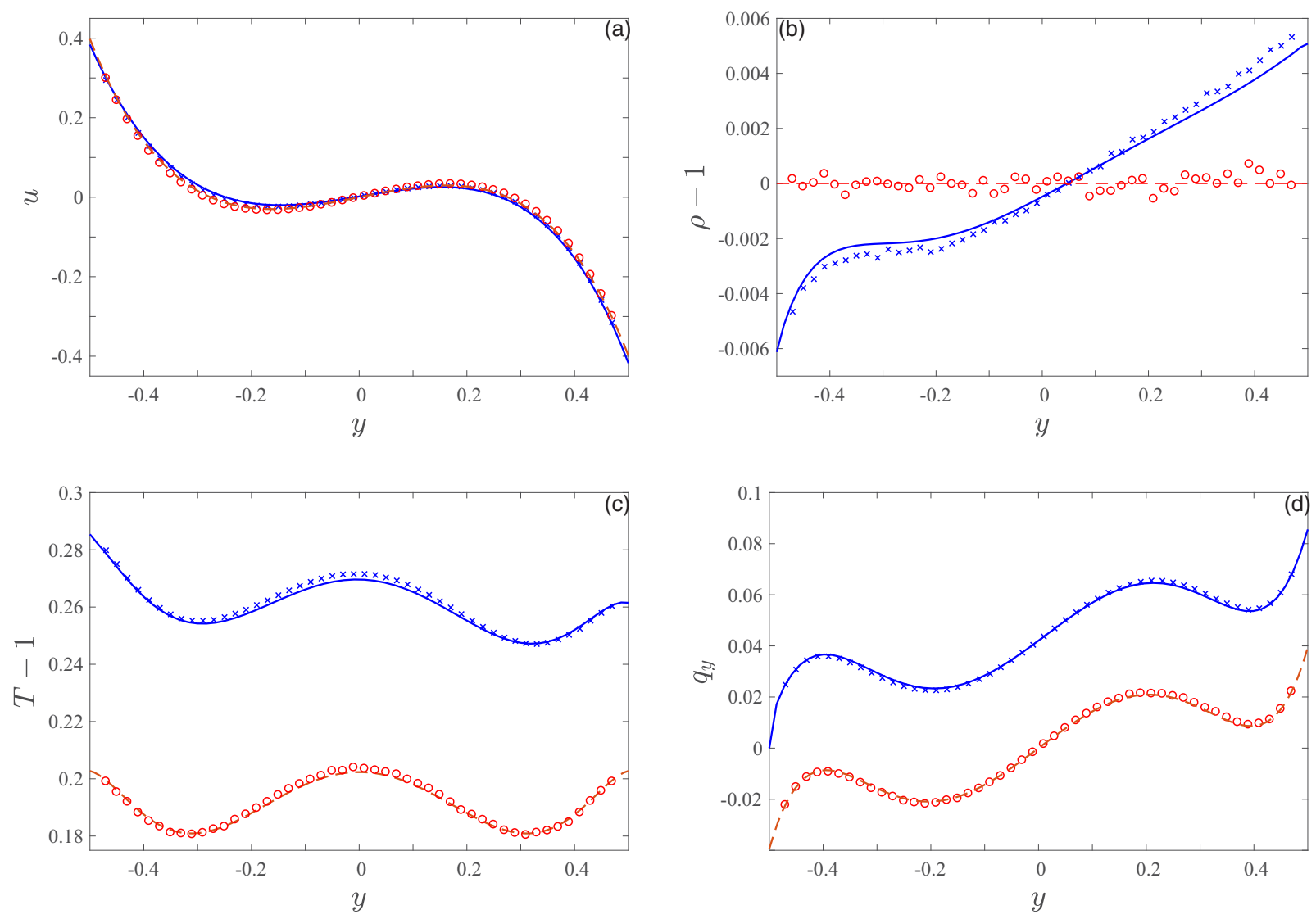

FIG. 2. Spatial variations of the (a) tangential velocity, (b) density deviation, (c) temperature deviation, and (d) normal heat flux, for the adiabatic wall (blue) and isothermal wall (red) setups with Ma $=0.8$ and $\omega=5$. The curves (solid blue and dashed red lines) and symbols (blue crosses and red circles) present the ballistic analytical and $\mathrm{Kn}=72 \mathrm{DSMC}$ predictions at period time, respectively.

impact on the tangential flow velocity. Indeed, all predictions in Fig. 3(a) coincide with the leading $O(\mathrm{Ma})$ slip-flow result, regardless of the applied thermal boundary conditions.

While the agreement between DSMC and slip-flow predictions appears reasonable, visible discrepancies are observed, particularly for the temperature deviation. This may be attributed to the relatively large value of $\mathrm{Kn}=0.09$ chosen, and perhaps to higher-order Mach corrections. Better agreement may be obtained by further decreasing $\mathrm{Kn}$. Yet, this must be followed by a subsequent decrease in the frequency $\omega$, so that $\omega \lesssim K n$, to prevent an increase in the local Knudsen number near the walls [see Eq. (32) et seq.], and associated magnification of the nonlinear forcing shear rate $\propto \omega / \mathrm{Kn}$ [see the forcing terms in Eqs. (40) and (41) together with Eq. (32)]. Since a further reduction in $\omega$ requires exceedingly long DSMC running times, such a comparison was not followed here.

To complement the description of the bidirectional flow field, Fig. 4 presents time snapshots of the normal velocity component $v(y, t)$. The high-Kn limit is presented in Fig. 4(a), where ballistic-flow predictions are compared with $\mathrm{Kn}=72$ DSMC results for $\mathrm{Ma}=0.8$ and $\omega=5$. The continuum limit is shown in Fig. 4(b), comparing DSMC and slip flow calculations for $\mathrm{Kn}=0.09, \mathrm{Ma}=0.3$, and $\omega=0.1$. Both adiabatic and isothermal wall setups are depicted at period time.
At first we note the relatively small amplitudes of the vertical velocity at both limits. Recalling the discussion of the ballistic regime in Figs. 1 and 2, nonzero normal velocity may occur in this limit only in the adiabatic wall setup, due to deviation of the adiabatic wall temperature from the reference equilibrium temperature. This is supported by the results in Fig. 4(a), showing a vanishing noisy DSMC signal in the isothermal case. This ballistic-regime restriction does not hold in the continuum limit in Fig. 4(b), where molecular collisions give rise to macroscopic normal velocity and momentum transfer regardless of the walls thermal conditions. In this limit, $\omega$ should be taken small, and $v(y, t)$ is consequently reduced due to mass balance considerations $(d v / d y \sim \omega \rho)$. The agreement between DSMC and slip flow predictions is good even within the Knudsen layers, presumably since the impermeability condition (in difference from the velocity slip and temperature jump conditions) is identically satisfied by both continuum limit and kinetic schemes.

Figures 5 and 6 present the tangential and normal stresses acting on the boundaries in an adiabatic wall setup. The figures show results for the stresses on both the adiabatic $y=-1 / 2$ and isothermal $y=1 / 2$ boundaries. Ballistic- and continuum-limit results are compared with DSMC predictions. Figure 5 describes the variations with $\mathrm{Kn}$ of the stresses amplitudes, demonstrating the breakdown of the limit-case analyses. Figure 6 depicts the variations with Ma, show- 

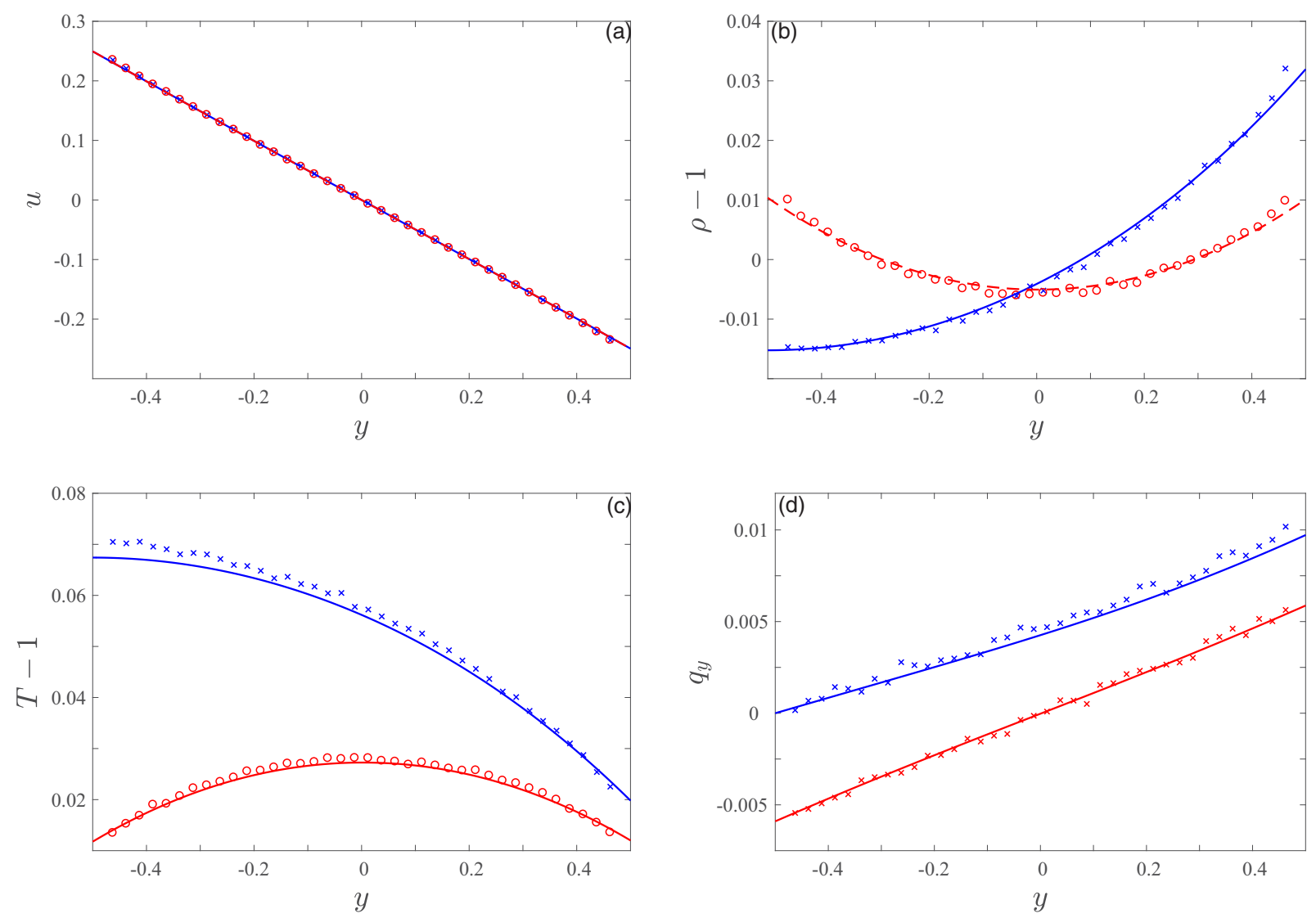

FIG. 3. Spatial variations of the (a) tangential velocity, (b) density deviation, (c) temperature deviation, and (d) normal heat flux, for the adiabatic wall (blue) and isothermal wall (red) setups with $\mathrm{Ma}=0.3, \omega=0.1$ and $\mathrm{Kn}=0.09$. The curves (solid blue and dashed red lines) and symbols (blue crosses and red circles) present slip flow analytical results and DSMC predictions at period time, respectively.

ing the effect of system nonlinearity at nonsmall Mach numbers.

Starting with the former, Fig. 5 describes the variations with $\mathrm{Kn}$ of the amplitudes of the tangential and normal walls stresses for $\omega=0.1$ with $\mathrm{Ma}=0.3$ [Figs. 5(a) and 5(b)], and $\mathrm{Ma}=0.8$ with $\omega=1$ [Figs. 5(c) and 5(d)]. DSMC results are compared with continuum and ballistic limit predictions at both walls. While it is expected that the nonlinear ballistic- flow calculation captures well the large-Kn wall stresses at arbitrary $\mathrm{Ma}$, the slip-flow scheme yields results that agree with DSMC calculations at Knudsen numbers larger than anticipated. This includes the $\mathrm{Ma}=0.8$ case, where $O\left(\mathrm{Ma}^{3}\right)$ corrections (not contained in the present calculation) could have become significant. Notably, visible discrepancies appear, in some of the cases, at low Knudsen numbers, and decrease with increasing $\mathrm{Kn}$. These are rationalized, as in
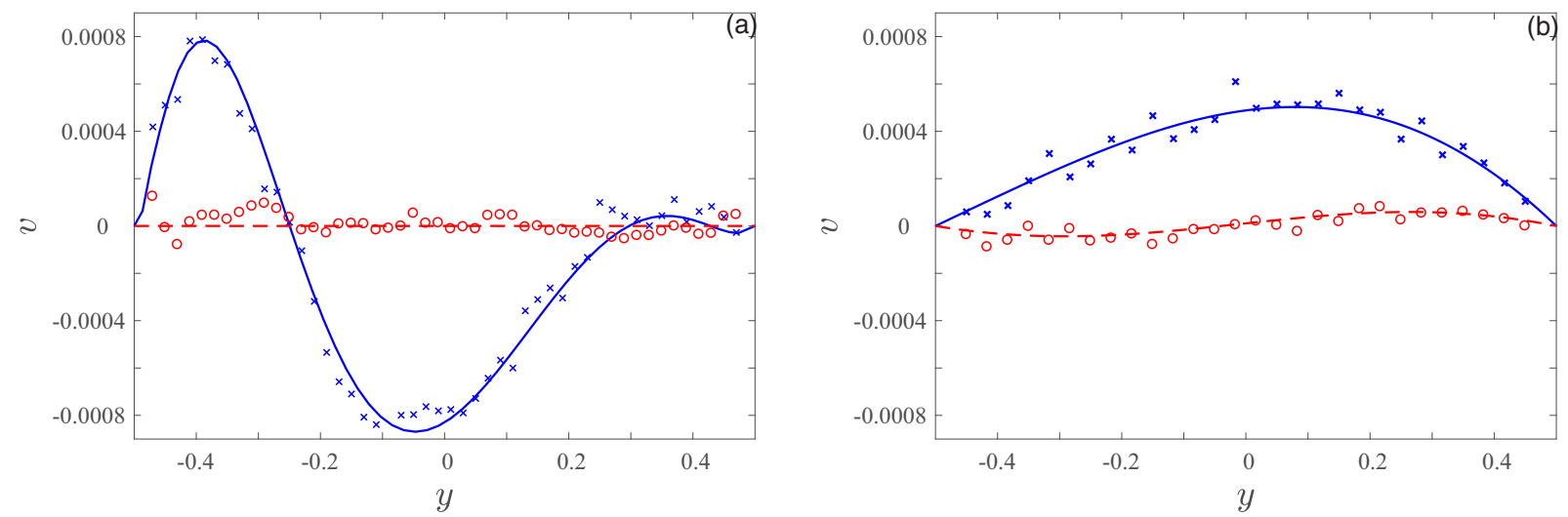

FIG. 4. Spatial variations of the normal velocity for the adiabatic wall (blue) and isothermal wall (red) setups with (a) Ma $=0.8, \omega=5$, and $\mathrm{Kn}=72$; (b) $\mathrm{Ma}=0.3, \omega=0.1$, and $\mathrm{Kn}=0.09$. The curves (solid blue and dashed red lines) and symbols (blue crosses and red circles) present analytical [collisionless in (a); slip flow in (b)] results and DSMC predictions at period time, respectively. 

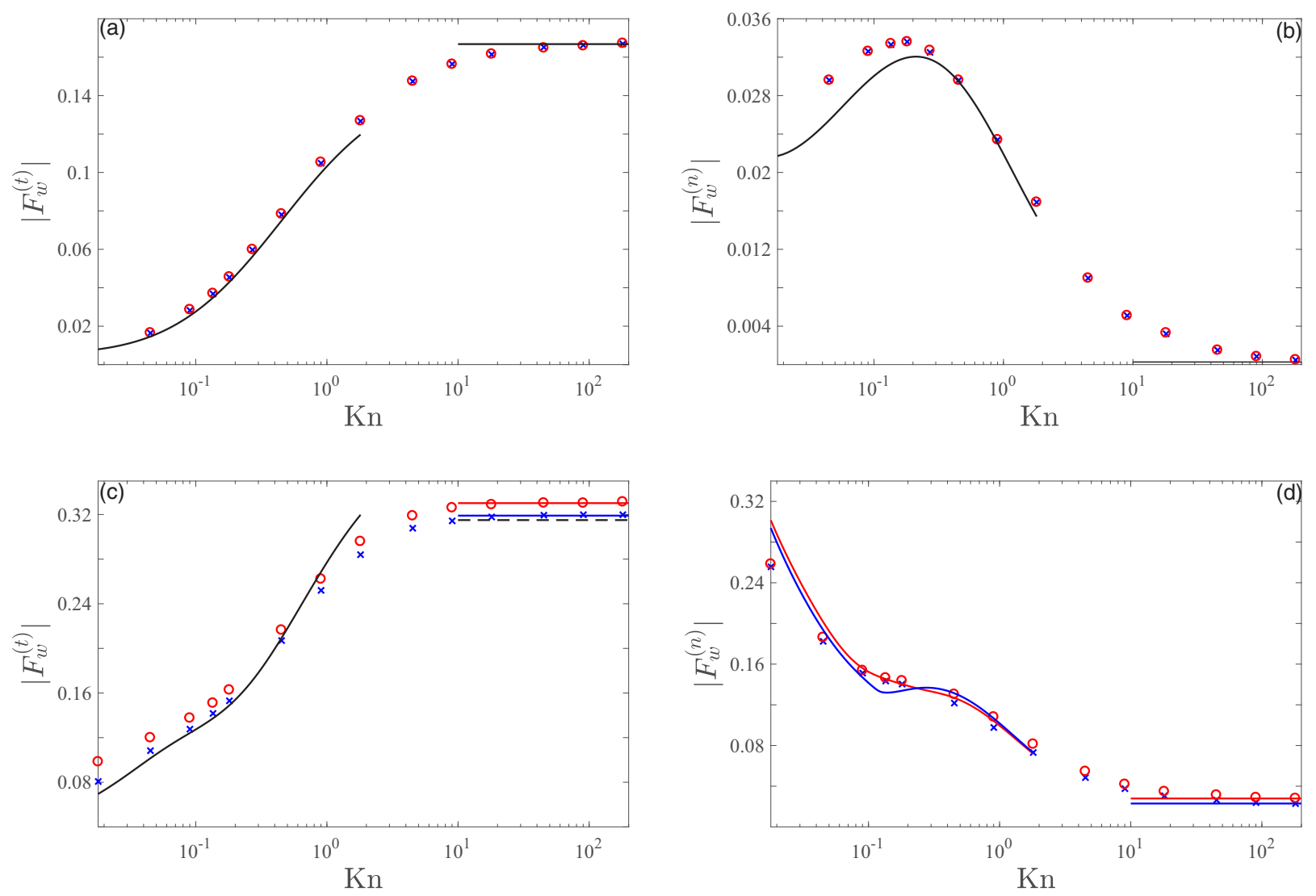

FIG. 5. Variations with $\mathrm{Kn}$ of the amplitudes of the (a, c) tangential and (b, d) normal forces per unit area on the adiabatic $y=-1 / 2$ (blue) and isothermal $y=1 / 2$ (red) boundaries for ( $\mathrm{a}, \mathrm{b}) \mathrm{Ma}=0.3$ and $\omega=0.1$; (c, d) Ma $=0.8$ and $\omega=1$. The symbols show DSMC results for the $y=-1 / 2$ (blue crosses) and $y=1 / 2$ (red circles) walls. The solid lines present continuum- and ballistic-limit predictions. Black curves are presented in cases where the adiabatic and isothermal walls results coincide. The black dashed line in (c) shows the linearized isothermal approximation for the tangential stress in the ballistic limit.

Fig. 3, by the respective increase in $\omega / \mathrm{Kn}$ for fixed $\omega$ and decreasing $\mathrm{Kn}$, which confines the walls-affected layers to the surfaces vicinities, magnifies the value of the local Knudsen number, and increases the associated nonlinear impact of gas shear rate [see Eq. (32) et seq.]. In view of the above, we find the overall agreement between the different schemes satisfactory, and in support of the validity of both calculations.

Comparing between the amplitudes of the tangential and normal stresses, we find that, for both Mach numbers, the tangential stress is considerably larger at high-Kn flow conditions
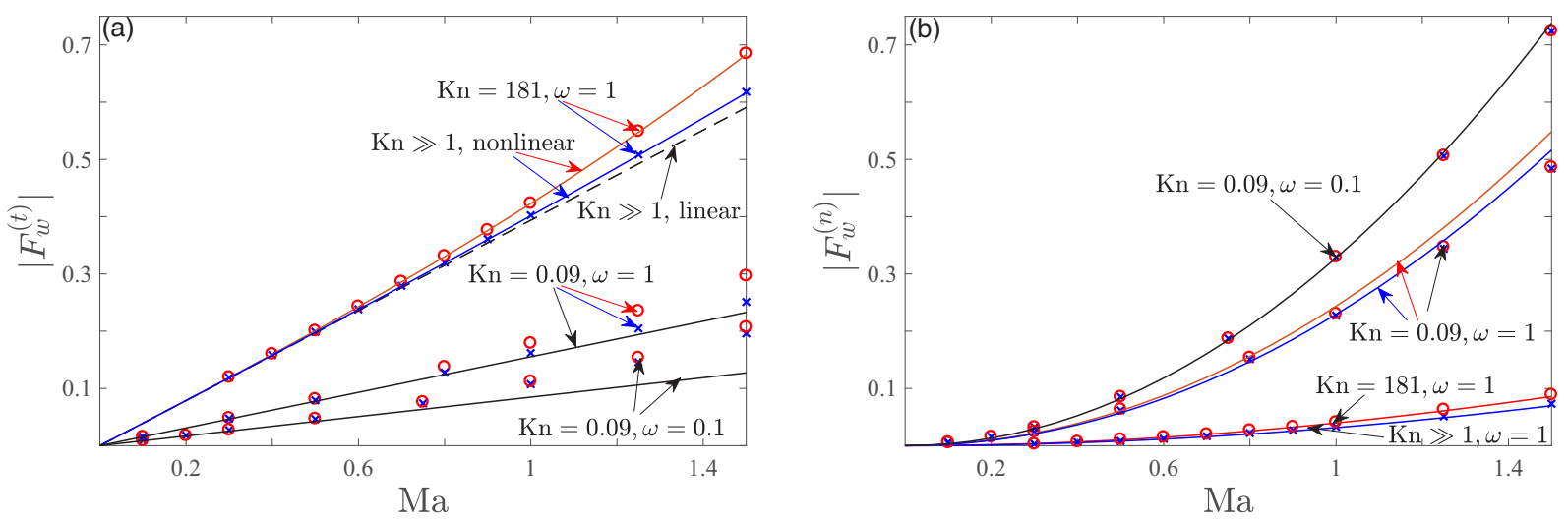

FIG. 6. Variations with Ma of the amplitudes of the (a) tangential and (b) normal forces per unit area on the adiabatic $y=-1 / 2$ (blue) and isothermal $y=1 / 2$ (red) boundaries at the indicated $(\mathrm{Kn}, \omega)$ combinations. The symbols show DSMC results for the $y=-1 / 2$ (blue crosses) and $y=1 / 2$ (red circles) walls. The solid lines present continuum- and ballistic-limit predictions. Black curves are presented where the adiabatic and isothermal walls results coincide. The black dashed line in (a) shows the linearized isothermal approximation for the tangential force in the ballistic limit at $\omega=1$. 

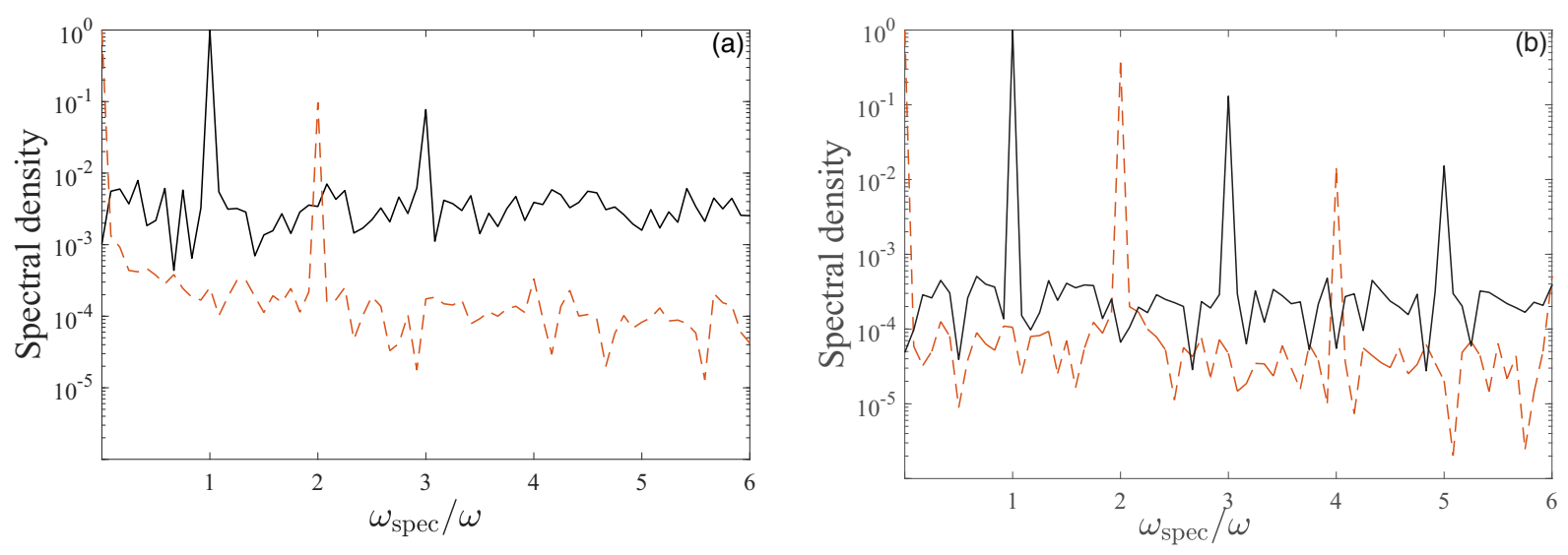

FIG. 7. Spectral densities of the tangential velocity (black solid lines) and temperature deviation (dashed red curves) signals at the gap middle point, $y=0$, for an adiabatic wall setup with $\mathrm{Kn}=0.09, \omega=1$ and (a) $\mathrm{Ma}=0.8$; (b) Ma $=5$. The spectra are calculated based on DSMC predictions and are scaled by their maximal values.

(cf. Fig. 6). Yet, at continuum-limit conditions, the normal stress, resulting from nonlinear thermal effects, becomes comparable with the tangential stress at $\mathrm{Ma}=0.3$, and overcomes with increasing Ma. Additionally, while the tangential force per unit area is always monotonically increasing with $\mathrm{Kn}$, the normal stress is either monotonically decreasing for $\omega \sim$ $O$ (1) [as for $\omega=1$ in Fig. 5(d)] or, at sufficiently low $\omega$, varies nonmonotonically and reaches a maximum at an intermediate Kn [as for $\omega=0.1$ in Fig. 5(b)]. In the following we seek to rationalize these findings.

The increase in $\left|F_{w}^{(t)}\right|$ with $\mathrm{Kn}$ has been observed in previous works studying the low-Ma isothermal problem (e.g., Ref. [15]). The tangential force on the wall is proportional to the difference in the tangential momentum between the incoming and reflected particles. In the high-Kn limit, the tangential momentum of a molecule impacting a wall at time $t^{*}$ depends almost entirely on the tangential velocity of the other wall at the "mean" retarded time $t^{*}-L^{*} / U_{\mathrm{th}}^{*}$. For the present opposite-phase motion of the boundaries, the two walls velocities are considerably different, resulting in significant momentum change due to boundary impact. With decreasing $\mathrm{Kn}$, collisions between the molecules become frequent, advancing inter-molecular momentum transfer during molecules passage between wall impacts. The tangential momentum change owing to particle-surface collisions is therefore reduced, and the walls shear stress reduces with $\mathrm{Kn}$. A similar conclusion may be drawn using macroscopic arguments, by examining the effect of decreasing the gap width $L^{*}$, while maintaining the gas reference properties $\left(\rho_{0}^{*}, T_{0}^{*}\right)$ and wall actuation parameters $\left(\bar{U}_{w}^{*}, \omega^{*}\right)$, on the shear stress amplitude $\left|F_{w}^{*(t)}\right|$. Assuming that the imposed shear rate is proportional to $\bar{U}_{w}^{*} / L^{*}$ (effective at small Knudsen numbers), it is clear that $\left|F_{w}^{*(t)}\right|$ increases with decreasing $L^{*}$. This is, again, in agreement with the results in Figs. 5(a) and 5(c) for $\left|F_{w}^{(t)}\right|$, which, when multiplying by the constant scaling factor $\rho_{0}^{*} U_{\text {th }}^{* 2}$, yield the increasing of $\left|F_{w}^{*(t)}\right|$ with $1 / L^{*}$.

Focusing on the low-Kn asymptotes in Figs. 5(a) and 5(c), we observe that a single curve appears, to approximate both adiabatic and isothermal wall tangential stresses. This is since, in the slip-flow calculation, only the leading $O(\mathrm{Ma})$ stress, unaffected by the wall thermal conditions, was evaluated. The
DSMC results for $\mathrm{Ma}=0.3$ in Fig. 5(a) support this approximation, showing no distinguishable differences between the shear stresses at the two walls. For the higher $\mathrm{Ma}=$ 0.8 presented in Fig. 5(c), the DSMC calculations predict higher tangential stress at the isothermal wall, which may be attributed to the $O\left(\mathrm{Ma}^{3}\right)$ correction not included in our calculation. The higher stress at the isothermal wall is retained at all Knudsen numbers, and is attributed to a thermal effect: molecules traversing from the adiabatic to the isothermal boundary acquire higher speed due to the larger temperature of the former. Hence, the momentum change at the isothermal wall impact is larger, resulting in a higher tangential stress.

Considering the ballistic-flow asymptotes in Figs. 5(a) and $5(\mathrm{c})$, we observe that for $\mathrm{Ma}=0.3$ the adiabatic and isothermal wall stresses coincide. At this combination of relatively low Mach number and frequency, the ballistic-flow tangential stress may be well approximated by the linearized isothermal result [obtained from Eqs. (15) and (17) in the limit $\rho_{w_{1,2}}=$ $\left.T_{w_{1}}=1\right]$,

$$
\left|F_{w_{1,2}}^{(t)}\right| \approx \mathrm{Ma} / \sqrt{\pi} \quad(\mathrm{Kn} \gg 1, \mathrm{Ma} \ll 1, \omega \ll 1),
$$

equal $\approx 0.169$ for $\mathrm{Ma}=0.3$. At $\mathrm{Ma}=0.8$, the linearized isothermal approximation [not captured by Eq. (64), since $\omega=1$ ] underestimates the ballistic-flow results, as visible by comparing between the dashed line and the solid high-Kn asymptotes in Fig. 5(c). These deviations, increasing with Ma, reflect the effects of system nonlinearity at large oscillation velocities, where increasing thermal dissipation rates couple elevated wall shear stresses.

Similar (yet qualitatively opposite) kinematic considerations may be used to rationalize the reversed trends observed for the variation with $\mathrm{Kn}$ of the normal stress amplitude $\left|F_{n}^{(t)}\right|$ in Figs. 5(b) and 5(d). At large-Kn conditions, molecular collisions are absent, and normal wall stresses are solely attributed to the deviations of the adiabatic wall temperature and gas medium from the reference temperature. These, in turn, cause an increase in the molecules normal velocity, which reflects in the relatively small (yet nonzero) values of $\left|F_{w_{1,2}}^{(n)}\right|$ in Figs. 5(b) and 5(d) at Kn $\gg 1$. The stresses increase with $\mathrm{Ma}$, in line with the larger deviation of the gas state from its reference isothermal condition at increasing velocities. 
Decreasing the value of $\mathrm{Kn}$ to intermediate $\mathrm{Kn} \lesssim O(1)$ values permits for molecular collisions to occur during the passage of molecules between the boundaries. Such collisions inevitably result in transferring molecular tangential momentum into normal momentum, with the change in the molecules postcollision normal velocity increasing for larger pre-collision tangential velocity differences. Inversely to the argument in the context of the tangential wall stress, this mechanism leads to the increase in the amplitude of the normal stress with decreasing $\mathrm{Kn}$.

Reducing the Knudsen number further to Kn $\ll 1$, molecular collisions become more frequent, with the mean free path shortening. When the oscillation frequency is small, the timescale for changes in the flow field is large, and it is therefore probable that collisions occur between molecules having similar tangential momenta. The post-collision difference in the molecules normal velocity is consequently smaller, and a reduction in the normal stress on the walls is observed. Importantly, this reduction is obtained particularly for $\omega \ll 1$, where the timescale characterizing the walls motion $\left(\sim 1 / \omega^{*}\right)$ is large compared with the scale characterizing molecules passage across the slab $\left(\sim L^{*} / U_{\mathrm{th}}^{*}\right)$. In such a case, the appearance of a maximum value for $\left|F_{w}^{(n)}\right|$ reflects a balance between the above-described counteracting effects: at some "optimal" value of $\mathrm{Kn} \lesssim 1$, the occurrence of few collisions during slab passage results in significant changes in molecules normal velocity, thus increasing the walls normal stress; below this "optimal" value, these changes become smaller owing to the common interactions between molecules having similar tangential momenta, and a decrease in $\left|F_{w}^{(n)}\right|$ is obtained.

The effect of the Mach number on the tangential and normal forces is considered in Fig. 6. Specifically, the variations of $\left|F_{w}^{(t)}\right|$ and $\left|F_{w}^{(n)}\right|$ with Ma are presented for low $(\mathrm{Kn}=0.09)$ and large $(\mathrm{Kn}=181)$ Knudsen numbers. The stresses on both adiabatic and isothermal walls are shown. DSMC results are compared with limit-case continuum and ballistic predictions at $\omega=0.1$ and $\omega=1$. At low $\mathrm{Kn}$, the Mach power expansion in Sec. IV predicts well the tangential linear [with $\sim O\left(\mathrm{Ma}^{3}\right)$ error] and normal quadratic [with $\sim O\left(\mathrm{Ma}^{4}\right)$ error] stress dependencies up to $\mathrm{Ma} \approx 0.8$. Beyond this value, the tangential and normal forces deviate from their analytic predictions due to higher-order effects. In marked difference, the nonlinear ballistic-flow analysis agrees well with the DSMC results for all Ma. For $\left|F_{w}^{(t)}\right|$, we observe a deviation between the nonlinear prediction and the linearized isothermal result at $\mathrm{Ma} \gtrsim 0.6$ [cf. the black dashed and solid blue and red curves in Fig. 6(a)]. While the tangential wall stress is invariably larger than the normal stress at large Kn, the latter overcomes $\left|F_{w}^{(t)}\right|$ for $\mathrm{Ma} \gtrsim 0.7$ in the continuum limit. This demonstrates the significance of system nonlinearity and thermal coupling on the shear-induced motion at nonsmall excitation velocities.

In terms of system time response, the results thus far have presented the effect of high-speed boundary actuation on the frequency doubling of the thermodynamic and normal velocity fields. While this is the main observation at finite yet small Mach numbers, closer inspection of the time signals at increasing Ma shows that amplified compressibility leads to the generation of higher-order harmonics in all hydrodynamic fields. To demonstrate that, Fig. 7 presents the
DSMC-calculated spectral densities of the tangential velocity and temperature signals at the gap middle point, $y=0$, for an adiabatic wall setup with $\mathrm{Kn}=0.09, \omega=1$ and different values of Ma. At the lower Ma =0.8 shown [Fig. 7(a)], the temperature field is dominated by the double frequency $\omega_{\text {spec }}=$ $2 \omega$ component, as supported by our continuum-limit analysis. Yet, the tangential velocity contains both the fundamental $\omega_{\text {spec }}=\omega$ and triple $\omega_{\text {spec }}=3 \omega$ frequency contributions. This trend becomes more pronounced at the higher $\mathrm{Ma}=5$ presented [Fig. 7(b)], where the tangential velocity contains an additional $\omega_{\text {spec }}=5 \omega$ contribution, and the temperature shows a secondary $\omega_{\text {spec }}=4 \omega$ peak. Generally, as the Mach number is increased at a given Knudsen, the solutions for the thermodynamic and normal velocity fields contain higher even harmonics (with $\omega_{\text {spec }}=2 n \omega, n=1,2, \ldots$ ), while the tangential velocity subsumes higher odd harmonics (with $\left.\omega_{\text {spec }}=(2 n+1) \omega, n=1,2, \ldots\right)$, both at descending amplitudes. Although higher-order corrections $\left(O\left(\mathrm{Ma}^{3}\right)\right.$ and above $)$ have not been calculated in the continuum-limit scheme, it is apparent through the asymptotic balance that such corrections would allow for multiple-harmonic components. According to our high-Kn DSMC and ballistic-limit spectra results (not presented here), the generation of higher harmonics turns quantitatively weaker with increasing $\mathrm{Kn}$.

\section{CONCLUSION}

We investigated the response of a rarefied gas in a slab to the motion of its boundaries in the tangential direction. In difference from previous studies, we considered boundaries displacements at nonsmall Mach numbers, coupling the dynamic and thermodynamic gas states, and deviating the system from its low-velocity isothermal condition. The problem was studied in the entire range of gas rarefaction conditions, combining limit case ballistic- and continuum-flow analyses with direct simulation Monte Carlo computations. Focusing on the case of oscillatory boundary motion, a nonlinear solution (also formulated for arbitrary wall-velocity profiles) was derived in the ballistic regime for arbitrary velocity amplitudes. At near-continuum conditions, a slip-flow time-periodic solution was obtained by expanding the flow field in a Mach power series. The effect of replacing between isothermal and adiabatic surfaces was examined. The results indicate that, at all Knudsen numbers, the thermodynamic fields and associated normal velocity component are dominated by doublefrequency (and descending higher-order even-frequency harmonic) time dependence, in difference from the fundamentalfrequency time dependence dominating the tangential gas velocity. At continuum-limit conditions, this stems from the quadratic viscous dissipation term appearing in the energy equation (negligible at low-Mach conditions), coupling the square of the tangential velocity gradient as a forcing term. System nonlinearity also results in an unsteady normal force acting on the boundaries, overcoming the tangential force at large enough Ma. In marked difference from the latter, the normal force either decreases with $\mathrm{Kn}$, or, at sufficiently small actuation frequencies, varies nonmonotonically with $\mathrm{Kn}$, reaching a maximum at some intermediate rarefaction conditions. 
Examining the impact of walls thermal boundary conditions, it was found that an adiabatic boundary, acting as a heat insulator, amplifies the effect of viscous dissipation in the slab, and results in elevated gas temperatures and heat fluxes. These, in turn, affect the gas-surface interactions, and modify the forces acting on the walls. Recalling the practical importance of controlling the shear and normal loads on the boundaries in a shear flow setup, it appears of interest to examine the possible monitoring of these forces by means of altering the walls-imposed heat flux. A study on this topic is currently in progress.

The present work considers a one-dimensional (bidirectional) slab setup, where all flow variables vary spatially with the normal coordinate only. A more general approach would examine a two-dimensional configuration, allowing for tangential-coordinate dependence of the flow field. Such an extension, which is not in the scope of the present contribution, may enable investigation of the stability of the one- dimensional solutions obtained here, similarly to previous continuum studies on shear flows [36]. In this context, we consider the present work as a preliminary step of obtaining a "reference" one-dimensional description. In view of previous analyses of the stability of rarefied gas flows in other canonical configurations (e.g., Refs. [37-39]), it appears reasonable to expect that gas rarefaction stabilizes the system state, thus confining instability phenomena (and associated two-dimensional effects) to the limit of small Knudsen numbers.

\section{ACKNOWLEDGMENT}

This research was supported by the Israel Science Foundation (Grant No. 1084/16). Y.B. is supported by the Adams Fellowship Program of the Israel Academy of Sciences and Humanities.

\section{APPENDIX A: EXPRESSIONS FOR THE HYDRODYNAMIC FIELDS IN THE BALLISTIC LIMIT}

The hydrodynamic density $\rho$, tangential velocity $u$, normal velocity $v$, pressure $p$, and normal heat flux $q_{y}$, obtained in the ballistic limit for arbitrary tangential wall excitation profiles $U_{w_{1,2}}(t)$, are

$$
\begin{aligned}
& \rho(y, t)=\frac{1}{\sqrt{\pi}} \int_{0}^{t}\left\{\frac{\rho_{w_{1}}(\tau)\left(y+\frac{1}{2}\right)}{T_{w_{1}}^{1 / 2}(\tau)} \exp \left[-\frac{\left(y+\frac{1}{2}\right)^{2}}{T_{w_{1}}(\tau)(t-\tau)^{2}}\right]\right. \\
& \left.-\rho_{w_{2}}(\tau)\left(y-\frac{1}{2}\right) \exp \left[-\frac{\left(y-\frac{1}{2}\right)^{2}}{(t-\tau)^{2}}\right]\right\} \frac{d \tau}{(t-\tau)^{2}}+\frac{1}{2}\left[\operatorname{erf}\left(\frac{y+\frac{1}{2}}{t}\right)-\operatorname{erf}\left(\frac{y-\frac{1}{2}}{t}\right)\right] \\
& u(y, t)=\frac{1}{\sqrt{\pi} \rho(y, t)} \int_{0}^{t}\left\{\frac{\rho_{w_{1}}(\tau) U_{w_{1}}(\tau)\left(y+\frac{1}{2}\right)}{T_{w_{1}}^{1 / 2}(\tau)} \exp \left[-\frac{\left(y+\frac{1}{2}\right)^{2}}{T_{w_{1}}(\tau)(t-\tau)^{2}}\right]\right. \\
& \left.-\rho_{w_{2}}(\tau) U_{w_{2}}(\tau)\left(y-\frac{1}{2}\right) \exp \left[-\frac{\left(y-\frac{1}{2}\right)^{2}}{(t-\tau)^{2}}\right]\right\} \frac{d \tau}{(t-\tau)^{2}} \\
& v(y, t)=\frac{1}{\sqrt{\pi} \rho(y, t)} \int_{0}^{t}\left\{\frac{\rho_{w_{1}}(\tau)\left(y+\frac{1}{2}\right)^{2}}{T_{w_{1}}^{1 / 2}(\tau)} \exp \left[-\frac{\left(y+\frac{1}{2}\right)^{2}}{T_{w_{1}}(\tau)(t-\tau)^{2}}\right]-\rho_{w_{2}}(\tau)\left(y-\frac{1}{2}\right)^{2}\right. \\
& \left.\times \exp \left[-\frac{\left(y-\frac{1}{2}\right)^{2}}{(t-\tau)^{2}}\right]\right\} \frac{d \tau}{(t-\tau)^{3}}+\frac{1}{2 \sqrt{\pi} \rho(y, t)}\left[\exp \left(-\frac{\left(y-\frac{1}{2}\right)^{2}}{t^{2}}\right)-\exp \left(-\frac{\left(y+\frac{1}{2}\right)^{2}}{t^{2}}\right)\right], \\
& p(y, t)=\frac{1}{3 \sqrt{\pi}} \int_{0}^{t}\left\{\frac{\rho_{w_{1}}(\tau)\left(y+\frac{1}{2}\right)}{T_{w_{1}}^{1 / 2}(\tau)}\left[T_{w_{1}}(\tau)+\left[U_{w_{1}}(\tau)-u(y, t)\right]^{2}+\left(\frac{y+\frac{1}{2}}{t-\tau}-v(y, t)\right)^{2}\right]\right. \\
& \times \exp \left[-\frac{\left(y+\frac{1}{2}\right)^{2}}{T_{1}(\tau)(t-\tau)^{2}}\right]-\rho_{w_{2}}(\tau)\left(y-\frac{1}{2}\right)\left[1+\left[U_{w_{2}}(\tau)-u(y, t)\right]^{2}+\left(\frac{y-\frac{1}{2}}{t-\tau}-v(y, t)\right)^{2}\right] \\
& \left.\times \exp \left[-\frac{\left(y-\frac{1}{2}\right)^{2}}{(t-\tau)^{2}}\right]\right\} \frac{d \tau}{(t-\tau)^{2}}+\frac{1}{6}\left\{\left(u^{2}(y, t)+v^{2}(y, t)+\frac{3}{2}\right)\left[\operatorname{erf}\left(\frac{y+\frac{1}{2}}{t}\right)-\operatorname{erf}\left(\frac{y-\frac{1}{2}}{t}\right)\right]\right. \\
& \left.+\frac{1}{\sqrt{\pi}}\left[\left(\frac{y-\frac{1}{2}}{t}-2 v(y, t)\right) \exp \left(-\left(\frac{y-\frac{1}{2}}{t}\right)^{2}\right)+\left(2 v(y, t)-\frac{y+\frac{1}{2}}{t}\right) \exp \left(-\left(\frac{y+\frac{1}{2}}{t}\right)^{2}\right)\right]\right\}
\end{aligned}
$$


and

$$
\begin{aligned}
q_{y}(y, t)= & \frac{1}{2 \sqrt{\pi}} \int_{0}^{t}\left\{\frac{\rho_{w_{1}}(\tau)}{T_{w_{1}}^{1 / 2}(\tau)}\left(y+\frac{1}{2}\right)\left(\frac{y+\frac{1}{2}}{t-\tau}-v(y, t)\right)\left[T_{w_{1}}(\tau)+\left[U_{w_{1}}(\tau)-u(y, t)\right]^{2}+\left(\frac{y+\frac{1}{2}}{t-\tau}-v(y, t)\right)^{2}\right]\right. \\
& \times \exp \left[-\frac{\left(y+\frac{1}{2}\right)^{2}}{T_{w_{1}}(\tau)(t-\tau)^{2}}\right]-\rho_{w_{2}}(\tau)\left(y-\frac{1}{2}\right)\left(\frac{y-\frac{1}{2}}{t-\tau}-v(y, t)\right)\left[1+\left[U_{w_{2}}(\tau)-u(y, t)\right]^{2}+\left(\frac{y-\frac{1}{2}}{t-\tau}-v(y, t)\right)^{2}\right] \\
& \left.\times \exp \left[-\frac{\left(y-\frac{1}{2}\right)^{2}}{(t-\tau)^{2}}\right]\right\} \frac{d \tau}{(t-\tau)^{2}}+\frac{1}{4 \sqrt{\pi}}\left\{\sqrt{\pi} v(y, t)\left(2 u^{2}(y, t)+v^{2}(y, t)+\frac{5}{2}\right)\left[\operatorname{erf}\left(\frac{y-\frac{1}{2}}{t}\right)-\operatorname{erf}\left(\frac{y+\frac{1}{2}}{t}\right)\right]\right. \\
& +\left[u^{2}(y, t)+3 v^{2}(y, t)+\frac{\left(y-\frac{1}{2}\right)^{2}}{t^{2}}-3 v(y, t) \frac{y-\frac{1}{2}}{t}+2\right] \exp \left[-\frac{\left(y-\frac{1}{2}\right)^{2}}{t^{2}}\right] \\
& \left.-\left[u^{2}(y, t)+3 v^{2}(y, t)+\left(\frac{y+\frac{1}{2}}{t}\right)^{2}-3 v(y, t) \frac{y+\frac{1}{2}}{t}+2\right] \exp \left[-\frac{\left(y+\frac{1}{2}\right)^{2}}{t^{2}}\right]\right\},
\end{aligned}
$$

respectively. In accordance with the equation of state and the scaling introduced, the temperature field is given by

$$
T(y, t)=2 p(y, t) / \rho(y, t) .
$$

\section{APPENDIX B: EXPRESSIONS FOR $\bar{v}^{(2)}(y)$ AND $\bar{\rho}^{(2)}(y)$ IN THE CONTINUUM-LIMIT SOLUTION}

The $2 \omega$-oscillatory amplitude functions of the $O\left(\mathrm{Ma}^{2}\right)$ vertical velocity $\bar{v}^{(2)}(y)$ and density $\bar{\rho}^{(2)}(y)$ are

$$
\begin{aligned}
& \bar{v}^{(2)}(y)=-\frac{\mathrm{i}}{2 \omega(\gamma-1)}\left\{\sqrt{\lambda_{1}}\left[B_{3} \exp \left(\sqrt{\lambda_{1}} y\right)-B_{1} \exp \left(-\sqrt{\lambda_{1}} y\right)\right]\left[k m \lambda_{1}-\left(\frac{1}{2}(\gamma-1)+2 \mathrm{i} \omega m\right)\right]\right. \\
& +\sqrt{\lambda_{2}}\left[B_{4} \exp \left(\sqrt{\lambda_{2}} y\right)-B_{2} \exp \left(-\sqrt{\lambda_{2}} y\right)\right]\left[k m \lambda_{2}-\left(\frac{1}{2}(\gamma-1)+2 \mathrm{i} \omega m\right)\right] \\
& +2 \sqrt{\frac{\mathrm{i} \omega}{\widetilde{\mathrm{Kn}}}}\left[C_{2} \exp \left(2 \sqrt{\frac{\mathrm{i} \omega}{\widetilde{\mathrm{Kn}}} y}\right)-C_{1} \exp \left(-2 \sqrt{\frac{\mathrm{i} \omega}{\widetilde{\mathrm{Kn}}}} y\right)\right]\left[4 \mathrm{i} m \frac{\omega \gamma}{\operatorname{Pr}}-\left(\frac{1}{2}(\gamma-1)+2 \mathrm{i} \omega m\right)\right] \\
& \left.+2 \mathrm{i} \omega m(\gamma-1) \sqrt{\frac{\mathrm{i} \omega}{\widetilde{\mathrm{Kn}}}}\left[A_{2}^{2} \exp \left(2 \sqrt{\frac{\mathrm{i} \omega}{\widetilde{\mathrm{Kn}}}} y\right)-A_{1}^{2} \exp \left(-2 \sqrt{\frac{\mathrm{i} \omega}{\widetilde{\mathrm{Kn}}}} y\right)\right]\right\}
\end{aligned}
$$

and

$$
\begin{aligned}
& \bar{\rho}^{(2)}(y)=\frac{1}{4 \omega^{2}(\gamma-1)}\left\{\lambda_{1}\left[B_{3} \exp \left(\sqrt{\lambda_{1}} y\right)+B_{1} \exp \left(-\sqrt{\lambda_{1}} y\right)\right]\left[k m \lambda_{1}-\left(\frac{1}{2}(\gamma-1)+2 \mathrm{i} \omega m\right)\right]\right. \\
& +\lambda_{2}\left[B_{4} \exp \left(\sqrt{\lambda_{2}} y\right)+B_{2} \exp \left(-\sqrt{\lambda_{2}} y\right)\right]\left[k m \lambda_{2}-\left(\frac{1}{2}(\gamma-1)+2 \mathrm{i} \omega m\right)\right] \\
& +4 \mathrm{i} \frac{\omega}{\widetilde{\mathrm{Kn}}}\left[C_{2} \exp \left(2 \sqrt{\frac{\mathrm{i} \omega}{\widetilde{\mathrm{Kn}}}} y\right)+C_{1} \exp \left(-2 \sqrt{\frac{\mathrm{i} \omega}{\widetilde{\mathrm{Kn}}}} y\right)\right]\left[4 \mathrm{i} m \frac{\omega \gamma}{\operatorname{Pr}}-\left(\frac{1}{2}(\gamma-1)+2 \mathrm{i} \omega m\right)\right] \\
& \left.-4 m(\gamma-1) \frac{\omega^{2}}{\widetilde{\mathrm{Kn}}}\left[A_{2}^{2} \exp \left(2 \sqrt{\frac{\mathrm{i} \omega}{\widetilde{\mathrm{Kn}}}} y\right)+A_{1}^{2} \exp \left(-2 \sqrt{\frac{\mathrm{i} \omega}{\widetilde{\mathrm{Kn}}}} y\right)\right]\right\} \text {, }
\end{aligned}
$$

respectively.

[1] F. M. White, Viscous Fluid Flow (McGraw-Hill, New York, 1991).
[2] L. Howarth, Some aspects of Rayleigh's problem for a compressible fluid, Q. J. Mech. Appl. Math. 4, 157 (1951). 
[3] M. Van Dyke, Impulsive motion of an infinite plate in a viscous compressible fluid, Z. Angew Math. Phys. 3, 343 (1952).

[4] K. Stewartson, On the motion of a flat plate at high speed in a viscous compressible fluid. I. Impulsive motion, Math. Proc. Cambridge Philos. Soc. 51, 202 (1955).

[5] M. Hanin, On Rayleigh's problem for compressible fluids, Q. J. Mech. Appl. Math. 13, 184 (1960).

[6] C. Cercignani and F. Sernagioto, The method of elementary solutions for time-dependent problems in linearized kinetic theory, Ann. Phys. 30, 154 (1964).

[7] Y. Sone, Kinetic theory analysis of linearized Rayleigh problem, J. Phys. Soc. Jpn. 19, 1463 (1964).

[8] L. Trilling, Asymptotic solution of the Boltzmann-Krook equation for the Rayleigh shear flow problem, Phys. Fluids 7, 1681 (1964).

[9] A. Beskok, G. E. Karniadakis, and W. Trimmer, Rarefaction and compressibility effects in gas micro flows, J. Fluids Eng. 118, 448 (1996).

[10] V. Garzo and A. Santos, Kinetic Theory of Gases in Shear Flows (Springer-Science+Business Media, Berlin, 2003).

[11] S. Stefanov, P. Gospodinov, and C. Cercignani, Monte Carlo simulation and Navier-Stokes finite difference calculation of unsteady-state rarefied gas flows, Phys. Fluids 10, 289 (1998).

[12] A. A. Abramov and A. V. Butkovskii, On the Rayleigh problem in the transitional regime: The sign change effect of the energy flux and other effects, Phys. Fluids 26, 077101 (2014).

[13] P. Bahukudumbi, J. H. Park, and A. Beskok, A unified engineering model for steady and quasi-steady shear-driven gas microflows, Microscale Thermophysical Engineering 7, 291 (2003).

[14] J. H. Park, P. Bahukudumbi, and A. Beskok, Rarefaction effects on shear driven oscillatory gas flows: A direct simulation Monte Carlo study in the entire Knudsen regime, Phys. Fluids 16, 317 (2004).

[15] N. G. Hadjiconstantinou, Oscillatory shear-driven gas flows in the transition and free-molecular-flow regimes, Phys. Fluids 17, 100611 (2005).

[16] F. Sharipov and D. Kalempa, Oscillatory Couette flow at arbitrary oscillation frequency over the whole range of the Knudsen number, Microfluid. Nanofluid. 4, 363 (2008).

[17] T. Doi, Numerical analysis of oscillatory Couette flow of a rarefied gas on the basis of the linearized Boltzmann equation for a hard sphere molecular gas, Z. Angew Math. Phys. 61, 811 (2010).

[18] Y. W. Yap and J. E. Sader, High accuracy numerical solutions of the Boltzmann Bhatnagar-Gross-Krook equation for steady and oscillatory Couette flows, Phys. Fluids 24, 032004 (2012).

[19] P. Gospodinov, V. Roussinov and S. Stefanov, Nonisothermal oscillatory cylindrical Couette gas flow in the slip regime: A computational study, Eur. J. Mech. B/Fluids 33, 14 (2012).

[20] L. Wu, J. M. Reese, and Y. Zhang, Oscillatory rarefied gas flow inside rectangular cavities, J. Fluid Mech. 748, 350 (2014).
[21] P. Wang, L. Zhu, W. Su, L. Wu, and Y. Zhang, Nonlinear oscillatory rarefied gas flow inside a rectangular cavity, Phys. Rev. E 97, 043103 (2018).

[22] R. D. Braun, M. A. Croom, J. S. Levine, and D. A. Spencer, Design of the ARES Mars airplane and mission architecture, J. Space. Rockets 43, 1026 (2006).

[23] E. Musk, Hyperloop Alpha, http://www.spacex.com/ sites/spacex/files/hyperloop_alpha-20130812.pdf (2011).

[24] J. Braun, J. Sousa, and C. Pekardan, Aerodynamic design and analysis of the Hyperloop, AIAA J. 55, 4053 (2017).

[25] M. M. J. Opgenoord and P. C. Caplan, On the aerodynamic design of the Hyperloop concept, Proceedings of the 35th AIAA Applied Aerodynamics Conference (AIAA, Denver, Colorado, 2017), Paper AIAA 2017-3740.

[26] C. Pekardan, and A. Alexeenko, Thermal lift generation and drag reduction in rarefied aerodynamics, in Proceedings of 30th International Symposium On Rarefied Gas Dynamics: RGD 30, AIP Conf. Proc. No. 1786 (AIP, Melville, 2016).

[27] C. Pekardan, and A. Alexeenko, Rarefaction effects for transonic airfoil flows at low Reynolds numbers, AIAA J. 56, 765 (2018).

[28] Y. Y. Kloss, D. V. Martynov, and F. G. Cheremisin, Computer simulation and analysis of the Holweck pump in the transient regime, Tech. Phys. 57, 451 (2012).

[29] S. Naris, C. Tantos, and D. Valuogeorgis, Kinetic modeling of a tapered Holweck pump, Vacuum 109, 341 (2014).

[30] K. E. Jousten, Handbook of Vacuum Technology (Wiley, New York, 2016).

[31] J. Iannacci, M. Huhn, C. Tschoban, and H. Potter, RFMEMS technology for future $(5 \mathrm{G})$ mobile and high-frequency applications: reconfigurable 8-bit power attenuator tested up to $110 \mathrm{GHz}$, IEEE Electron Device Lett. 37, 1646 (2016).

[32] Y. Sone, Molecular Gas Dynamics: Theory, Techniques, and Applications (Birkhäuser, Boston, 2007).

[33] K. Aoki, C. Baranger, M. Hattori, S. Kosuge, G. Martalo, J. Mathiaud, and L. Mieussens, Slip boundary conditions for the compressible Navier-Stokes equations, J. Stat. Phys. 169, 744 (2017).

[34] G. Bird, Molecular Gas Dynamics and the Direct Simulation of Gas Flows (Clarendon, Oxford, 1994).

[35] Y. Ben Ami and A. Manela, Acoustic field of a pulsating cylinder in a rarefied gas: Thermoviscous and curvature effects, Phys. Rev. Fluids 2, 093401 (2017).

[36] P. J. Schmid and D. S. Henningson, Stability and Transition in Shear Flows (Springer, Berlin, 2001).

[37] S. Stefanov, V. Roussinov, and C. Cercignani, Rayleigh-Benard flow of a rarefied gas and its attractors. I. Convection regime, Phys. Fluids 14, 2255 (2002).

[38] M. Manela and I. Frankel, On the compressible Taylor-Couette problem, J. Fluid Mech. 588, 59 (2007).

[39] M. Manela and J. Zhang, The effect of compressibility on the stability of wall-bounded Kolmogorov flow, J. Fluid Mech. 694, 29 (2012). 